\title{
Axiomatic quantum field theory in curved spacetime
}

\author{
Stefan Hollands ${ }^{a *}$, Robert M. Wald ${ }^{\dagger \dagger}$ \\ ${ }^{a}$ School of Mathematics, Cardiff University, $U K$ \\ ${ }^{b}$ Enrico Fermi Institute and Department of Physics \\ University of Chicago, Chicago, IL, USA
}

March 13, 2008

\begin{abstract}
The usual formulations of quantum field theory in Minkowski spacetime make crucial use of features - such as Poincare invariance and the existence of a preferred vacuum state - that are very special to Minkowski spacetime. In order to generalize the formulation of quantum field theory to arbitrary globally hyperbolic curved spacetimes, it is essential that the theory be formulated in an entirely local and covariant manner, without assuming the presence of a preferred state. We propose a new framework for quantum field theory, in which the existence of an Operator Product Expansion (OPE) is elevated to a fundamental status, and, in essence, all of the properties of the quantum field theory are determined by its OPE. We provide general axioms for the OPE coefficients of a quantum field theory. These include a local and covariance assumption (implying that the quantum field theory is locally and covariantly constructed from the spacetime metric), a microlocal spectrum condition, an "associativity" condition, and the requirement that the coefficient of the identity in the OPE of the product of a field with its adjoint have positive scaling degree. We prove curved spacetime versions of the spin-statistics theorem and the PCT theorem. Some potentially significant further implications of our new viewpoint on quantum field theory are discussed.
\end{abstract}

*HollandsS@Cardiff.ac.uk

†rmwa@midway.uchicago.edu 


\section{Introduction}

The Wightman axioms [27] of quantum field theory in Minkowski spacetime are generally believed to express the fundamental properties that quantum fields possess. In essence, these axioms require that the following key properties hold: (1) The states of the theory are unit rays in a Hilbert space, $\mathcal{H}$, that carries a unitary representation of the Poincare group. (2) The 4-momentum (defined by the action of the Poincare group on the Hilbert space) is positive, i.e., its spectrum is contained within the closed future light cone ("spectrum condition"). (3) There exists a unique, Poincare invariant state ("the vacuum"). (4) The quantum fields are operator-valued distributions defined on a dense domain $\mathcal{D} \subset \mathcal{H}$ that is both Poincare invariant and invariant under the action of the fields and their adjoints. (5) The fields transform in a covariant manner under the action of Poincare transformations.

(6) At spacelike separations, quantum fields either commute or anticommute.

During the past 40 years, considerable progress has been made in understanding both the physical and mathematical properties of quantum fields in curved spacetime. Although gravity itself is treated classically, this theory incorporates some key aspects of general relativity and thereby should provide a more fundamental base for quantum field theory. Much of the progress has occurred in the analysis of free (i.e., non-self-interacting) fields, but in the past decade, major progress also has been made in the perturbative analysis of interacting quantum fields. Significant insights have thereby been obtained into the nature of quantum field phenomena in strong gravitational fields. In addition, some important insights have been obtained into the nature of quantum field theory itself. One of the key insights is that - apart from stationary spacetimes or spacetimes with other very special properties - there is no unique, natural notion of a "vacuum state" or of "particles". Indeed, unless the spacetime is asymptotically stationary at early or late times, there will not, in general, even be an asymptotic notion of particle states. Consequently, it is essential that quantum field theory in curved spacetime be formulated in terms of the local field observables as opposed, e.g., to S-matrices.

Since quantum field theory in curved spacetime should be much closer to a true theory of nature than quantum field theory in Minkowski spacetime, it is of interest to attempt to abstract the fundamental features of quantum field theory in curved spacetime in a manner similar to the way the Wightman axioms abstract what are generally believed to be the fundamental features of quantum field theory in Minkowski spacetime. The Wightman axioms are entirely compatible with the focus on local field observables, as needed for a formulation of quantum field theory in curved spacetime. However, most of the properties of quantum fields stated in the Wightman axioms are very special to Minkowski spacetime and cannot be generalized straightforwardly to curved spacetime. Specifically, a curved spacetime cannot possess Poincare symmetry-indeed a generic curved spacetime will not possess any symmetries at all—so one certainly cannot require "Poincare invariance/covariance" or invariance under any other type of spacetime symmetry. Thus, no direct analog of properties (3) and (5) can be imposed in curved spacetime, and the key aspects of properties (1) and (2) (as well as an important aspect of (4)) also do not make sense. 
In fact, the situation with regard to importing properties (1), (2), and (4) to curved spacetime is even worse than would be suggested by merely the absence of symmetries: There exist unitarily inequivalent Hilbert space constructions of free quantum fields in spacetimes with a noncompact Cauchy surface and (in the absence of symmetries of the spacetime) none appears "preferred". Thus, it is not appropriate even to assume, as in (1), that states are unit rays in a single Hilbert space, nor is it appropriate to assume, as in (4), that the (smeared) quantum fields are operators on this unique Hilbert space. With regard to (2), although energy and momentum in curved spacetime cannot be defined via the action of a symmetry group, the stress-energy tensor of a quantum field in curved spacetime should be well defined as a distributional observable on spacetime, so one might hope that it might be possible to, say, integrate the (smeared) energy density of a quantum field over a Cauchy surface and replace the Minkowski spacetime spectrum condition by the condition that the total energy of the quantum field in any state always is non-negative. However, this is not a natural thing to do, since the "total energy" defined in this way is highly slice/smearing dependent, and it is well known in classical general relativity that in asymptotically flat spacetimes, the integrated energy density of matter may bear little relationship to the true total mass-energy. Furthermore, it is well known that the energy density of a quantum field (in flat or curved spacetime) can be negative, and, in some simple examples involving free fields in curved spacetime, the integrated energy density is found to be negative. Consequently, there is no analog of property (2) in curved spacetime that can be formulated in terms of the "total energy-momentum" of the quantum field. Thus, of all of the properties of quantum fields in Minkowski spacetime stated in the Wightman axioms, only property (6) has a straightforward generalization to curved spacetimes!

Nevertheless, it has been understood for quite some time that the difficulties in the formulation of quantum field theory in curved spactime that arise from the existence of unitarily inequivalent Hilbert space constructions of the theory can be overcome by simply formulating the theory via the algebraic framework [14]. Instead of starting from the postulate that the states of the theory comprise a Hilbert space and that the (smeared) quantum fields are operators on this Hilbert space, one starts with the assumption that the (smeared) quantum fields (together with the identity element $\mathbf{1}$ ) generate a $*$-algebra, $\mathcal{A}$. States are then simply expectation functionals $\langle.\rangle_{\omega}: \mathcal{A} \rightarrow \mathbb{C}$ on the algebra, i.e., linear functionals that are positive in the sense that $\left\langle A^{*} A\right\rangle_{\omega} \geq 0$ for all $A \in \mathcal{A}$. The GNS construction then assures us that given a state, $\omega$, one can find a Hilbert space $\mathcal{H}$ that carries a representation, $\pi$, of the $*_{\text {-algebra }} \mathcal{A}$, such that there exists a vector $|\Psi\rangle \in \mathcal{H}$ for which $\langle A\rangle_{\omega}=\langle\Psi|\pi(A)| \Psi\rangle$ for all $A \in \mathcal{A}$. All of the operators, $\pi(A)$, are automatically defined on a common dense invariant domain, $\mathcal{D} \subset \mathcal{H}$, and each vector $|\Psi\rangle \in \mathcal{D}$ defines a state via $\langle A\rangle_{\Psi}=\langle\Psi|\pi(A)| \Psi\rangle$. Thus, by simply adopting the algebraic viewpoint, we effectively incorporate into quantum field theory in curved spacetime the portions of the content of properties (1) and (4) above that do not refer to Poincare symmetry.

It is often said that in special relativity one has invariance under "special coordinate transformations" (i.e., Poincare transformations), whereas in general relativity, one has invariance under "general coordinate transformations" (i.e., all diffeomorphisms). Thus, one might be tempted to think that the Minkowski spacetime requirements of invariance/covariance 
under Poincare transformations could be generalized to curved spacetime by requiring a similar "invariance/covariance under arbitrary diffeomorphisms". However, such thoughts are based upon a misunderstanding of the true meaning of "special covariance" and "general covariance". By explicitly incorporating the flat spacetime metric, $\eta_{a b}$, into the formulation of special relativity, it can easily be seen that special relativity can be formulated in as "generally covariant" a manner as general relativity. However, the act of formulating special relativity in a generally covariant manner does not provide one with any additional symmetries or other useful conditions on physical theories in flat spacetime. The point is that in special relativity, Poincare transformations are symmetries of the spacetime structure, and we impose a nontrivial requirement on a physical theory when we demand that its formulation respect these symmetries. However, a generic curved spacetime will not possess any symmetries at all, so no corresponding conditions on a physical theory can be imposed. The demand that a theory be "generally covariant" (i.e., that its formulation is invariant under arbitrary diffeomorphisms) can always be achieved by explicitly incorporating any "background structure" into the formulation of the theory. If one considers a fixed, curved spacetime without symmetries, no useful conditions can be imposed upon a quantum field theory by attempting to require some sort of "invariance" of the theory under diffeomorphisms.

However, there is a meaningful notion of "general covariance" that can be very usefully and powerfully applied to quantum field theory in curved spacetime. The basic idea behind this notion is that the only "background structure" that should occur in the theory is the spacetime manifold and metric modulo diffeomorphisms, together with the time and space orientations and (if spinors are present in the theory) spin structure. The quantum fields should be "covariant" in that they should be constructed from only this background structure. Indeed, since the smeared quantum fields are associated with local regions of spacetime (namely, the support of the test function used for the smearing), it seems natural to demand that the quantum fields be locally constructed from the background structure in the sense that the quantum fields in any neighborhood $O$ be covariantly constructed from the background structure within $O$. This idea may be formulated in a precise manner as follows [19, 20, 7].

First, in order to assure a well defined dynamics and in order to avoid causal pathologies, we restrict consideration to globally hyperbolic spacetimes $\left(M, g_{a b}\right)$. (We consider theories in arbitrary spacetime dimension $D \equiv \operatorname{dim} M \geq 2$.) If spinors are present in the theory, we also demand that $M$ admit a spin structure. It is essential that the quantum field theory be defined on all $D$-dimensional globally hyperbolic spacetimes admitting a spin structure, since in essence, we can only tell whether the quantum field is "locally and covariantly constructed out of the metric" if we can see how the theory changes when we change the metric in an arbitrary way. The "background structure", $\mathbf{M}$, of the theory is taken to consist of the manifold $M$, the metric $g_{a b}$, the spacetime orientation-which may be represented by a nowhere vanishing $D$-form, $e_{a_{1} \ldots a_{D}}$ on $M$-and a time orientation-which may be represented e.g. by the equivalence class of a time function $T: M \rightarrow \mathbb{R}$-i.e., we have

$$
\mathbf{M}=(M, g, T, e) .
$$


(If spinors are present in the theory, and $M$ admits more than one spin structure, then the choice of spin-structure over $M$ also should be understood to be included M.) For each choice of $\mathbf{M}$, we assume that there is specified a *algebra $\mathcal{A}(\mathbf{M})$ that is generated by a countable list of quantum fields $\phi^{(i)}$ and their "adjoints" $\phi^{(i) *}$. These fields may be of arbitrary tensorial or spinorial type, and they are smeared with arbitrarily chosen smooth, compact support fields of dual tensorial or spinorial type. In order to determine if the quantum field theory and quantum fields $\phi^{(i)}$ are "locally and covariantly constructed out of the background structure $\mathbf{M}^{\prime}$, we consider the following situation: Let $(M, g)$ and $\left(M^{\prime}, g^{\prime}\right)$ be two globally hyberbolic spacetimes that have the property that there exists a one-to-one (but not necessarily onto) map $\rho: M \rightarrow M^{\prime}$ that preserves all of the background structure. In other words, $\rho$ is an isometric imbedding that is orientation and time orientation preserving (and, if spinors are present, the choices of spin structure on $M$ and $M^{\prime}$ correspond under $\rho$ ). We further assume that $\rho$ is causality preserving in the sense that if $x_{1}, x_{2} \in M$ cannot be connected by a causal curve in $M$, then $\rho\left(x_{1}\right)$ and $\rho\left(x_{2}\right)$ cannot be connected by a causal curve in $M^{\prime}$. We say that the quantum field theory is locally and covariantly constructed from $\mathbf{M}$ (or, for short, that the theory is local and covariant) if (i) for every such $\mathbf{M}, \mathbf{M}^{\prime}$, and $\rho$ we have a corresponding *-isomorphism $\chi_{\rho}$ between $\mathcal{A}(\mathbf{M})$ and the subalgebra of $\mathcal{A}\left(\mathbf{M}^{\prime}\right)$ generated by the quantum fields $\phi^{(i)}$ and $\phi^{(i) *}$ smeared with test fields with support in $\rho[M]$ and (ii) if $\rho^{\prime}$ is a similar background structure and causality preserving map taking $\mathbf{M}^{\prime}$ to $\mathbf{M}^{\prime \prime}$, then $\chi_{\rho^{\prime} \circ \rho}=\chi_{\rho^{\prime}} \circ \chi_{\rho}$. We further say that the quantum field $\phi^{(i)}$ is locally and covariantly constructed from $\mathbf{M}$ (or, for short, that $\phi^{(i)}$ is local and covariant) if for every such $\mathbf{M}, \mathbf{M}^{\prime}$, and $\rho$, we have

$$
\chi_{\rho}\left(\phi^{(i)}(f)\right)=\phi^{(i)}\left(\rho_{*}(f)\right),
$$

where $\rho_{*}(f)$ denotes the natural push-forward action of $\rho$ on the tensor/spinor field $f$ on $M$.

Note that in contrast to the notion of Poincare invariance/covariance-which applies to quantum field theory on a single spacetime (namely, Minkowski spacetime)- the notion that a quantum field theory or quantum field is local and covariant is a condition that applies to the formulation of quantum field theory on different spacetimes. Nevertheless, the close relationship between these notions can be seen as follows: Suppose that we have a local and covariant quantum field theory, with local and covariant quantum fields $\phi^{(i)}$. Let $\mathbf{M}$ and $\mathbf{M}^{\prime}$ both be the background structure of Minkowski spacetime, and let $\rho$ be a proper Poincare transformation. Then $\rho$ preserves all of the background structure, so for each proper Poincare transformation, we obtain a $*$-isomorphism $\chi_{\rho}: \mathcal{A} \rightarrow \mathcal{A}$, where $\mathcal{A}$ here denotes the quantum field algebra for Minkowski spacetime. Furthermore, if $\rho$ and $\rho^{\prime}$ are proper Poincare transformations, we have $\chi_{\rho \circ \rho^{\prime}}=\chi_{\rho} \circ \chi_{\rho^{\prime}}$. Thus, every local and covariant quantum field theory in curved spacetime gives rise to a Poincare invariant theory in this sense when restricted to Minkowski spacetime. Furthermore, if $\phi^{(i)}$ is a local and covariant quantum field, then in Minkowski spacetime it transforms covariantly via eq.(2) under proper Poincare transformations.

Note also that, more generally, in any curved spacetime with symmetries, a local and covariant quantum field theory will be similarly invariant under these symmetries, and a 
local and covariant quantum field will transform covariantly under these symmetries. But even for spacetimes without any symmetries, the requirement that the quantum field theory and quantum fields be local and covariant imposes a very powerful restriction akin to requiring Poincare invariance/covariance in Minkowski spacetime.

From these considerations, it can be seen that if we adopt the above algebraic framework for quantum field theory in curved spacetime and if we additionally demand that the quantum field theory and the quantum fields $\phi^{(i)}$ be local and covariant, then we obtain satisfactory generalizations to curved spacetime of properties (1), (4), and (5) of the Wightman axioms in Minkowski spacetime. Since we already noted that (6) has a trivial generalization to curved spacetime, only properties (2) and (3) remain to be generalized to curved spacetime.

We have already noted above that there is no analog of property (2) in curved spacetime that can be formulated in terms of the "total energy-momentum" of the quantum field. However, it is possible to reformulate the spectrum condition in Minkowski spacetime in terms of purely local properties of the quantum fields. Specifically, the "positive frequency" (and, thereby, positive energy) properties of states are characterized by the short-distance singularity structure of the $n$-point functions of the quantum fields, as described by their wavefront set. One thereby obtains a microlocal spectrum condition [24, 5, 6] that is formulated purely in terms of the local in spacetime properties of the quantum fields. This microlocal spectrum condition has a natural generalization to curved spacetime (see section 2 below), thus providing the desired generalization of property (2) to curved spacetime.

Consequently, only property (3) remains to be generalized. In Minkowski spacetime, the existence of a unique, Poincare invariant state has very powerful consequences, so it is clear that a key portion of the content of quantum field theory in Minkowski spacetime would be missing if we failed to impose an analogous condition in curved spacetime. However, as already mentioned above, one of the clear lessons of the study of free quantum fields in curved spacetime is that, in a general curved spacetime, there does not exist a unique, "preferred" vacuum or other state. Furthermore, even if a prescription for finding a unique "preferred state" on each spacetime could be found, since generic curved spacetimes do not have any symmetries and states on different spacetimes cannot be meaningfully compared, there would appear to be no sensible "invariance" properties that such a preferred state could have. We do not believe that property (3) can be generalized to curved spacetime by a condition that postulates the existence of a preferred state with special properties.

In addition, we question the fundamental status of demanding the existence of a state that is invariant under the symmetries of the spacetime. For example, it is well known that the free massless Klein-Gordon field in two-dimensional Minkowski spacetime does not admit a Poincare invariant state. However, there is absolutely nothing wrong with the quantum field algebra of this field; the quantum field theory is "Poincare invariant" and the quantum field is "Poincare covariant" in the sense described above. Furthermore, there is no shortage of physically acceptable ("Hadamard") states. Thus, the only thing unusual about this quantum field theory is that it happens not to admit a Poincare invariant state. We do not feel that this is an appropriate reason to exclude the free massless Klein-Gordon field in two-dimensional Minkowski from being considered to be a legitimate quantum field 
theory. Similar remarks apply to the free Klein-Gordon field of negative $m^{2}$ in Minkowski spacetime of all dimensions. The classical and quantum dynamics of this field are entirely well posed and causal, although they are unstable in the sense of admitting solutions/states where the field grows exponentially with time. This instability provides legitimate grounds for arguing that the free Klein-Gordon field of negative $m^{2}$ does not occur in nature, but we do not feel that the absence of a Poincare invariant state constitutes legitimate grounds for rejecting this theory as a quantum field theory; see section 6 below for further discussion.

For the above reasons, we seek a replacement of property (3) that does not require the existence of states of a special type. The main purpose of this paper is to propose that the appropriate replacement of property (3) for quantum field theory in curved spacetime is to postulate the existence of a suitable operator product expansion [29, 31, 26] of the quantum fields. The type of operator product expansion that we shall postulate is known to hold in free field theory and to hold order by order in peturbation theory on any Lorentzian curved spacetime [15]. We thus propose to elevate this operator product expansion to the status of a fundamental property of quantum fields1. Although the assumption of the existence of an operator product expansion in quantum field theory in curved spacetime is remarkably different in nature from the assumption of the existence of a Poincare invariant state in quantum field theory in Minkowski spacetime, we will show in sections 4 and 5 below that it can do "much of the same work" as the latter assumption. It is shown in [21] how to exploit consistency conditions on the OPE in a framework closely related to that presented here. In particular, it is shown how perturbations of a quantum field theory can be characterized and calculated via consistency conditions arising from the OPE.

We have an additional motivation for proposing to elevate the operator product expansion to the status of a fundamental property of quantum fields. For free quantum fields in curved spacetime, an entirely satisfactory $*_{\text {-algebra, }} \mathcal{A}_{0}$, of observables has been constructed [5, 6, 19], which includes all Wick powers and time-ordered products. However, the elements of $\mathcal{A}_{0}$ correspond to unbounded operators, and there does not seem to be any natural algebra of bounded elements (with, e.g., a $C^{*}$-structure) corresponding to $\mathcal{A}_{0}$. Furthermore, $\mathcal{A}_{0}$ does not appear to have any natural topology (apart from a topology that can be defined a postiori by using the allowed states as semi-norms). Fortunately, a topology is not actually needed to define $\mathcal{A}_{0}$ because the relations that hold in $\mathcal{A}_{0}$ can be expressed in terms of finite sums of finite products of fields. However, it is inconceivable that the relations that define an interacting field algebra will all be expressible in terms of finite sums of finite products of the fields. Thus, without a natural topology and without finitely expressible relations, it is far from clear as to how an interacting field algebra might be defined. We claim that an operator-product expansion effectively provides the needed "relations" between the quantum fields, and we will propose in this paper that these relations are sufficient to define a quantum field theory. In other words, we believe that an interact-

\footnotetext{
${ }^{1}$ Various axiomatic approaches to conformal field theories based on the operator product expansion have been proposed previously, see e.g. the one based upon the notion of "vertex operator algebras" given in [2, 23, 11]. However, in contrast to our approach, these approaches incorporate in an essential way the conformal symmetry of the underlying space. In some approaches to quantum field theory on Minkowski space, the OPE is not postulated, but instead derived [10, 3, 4].
} 
ing quantum field theory is, in essence, defined via its operator-product expansion. From this perspective, it seems natural to view the operator product-expansion as a fundamental aspect of the quantum field theory.

In the next section, we will describe our framework for quantum field theory in curved spacetime. In particular, we will provide a precise statement of the what we mean by an operator-product expansion and the properties that we will assume that it possesses. In section 3, we will state our axioms for quantum field theory in curved spacetime and explain how it is constructed from the operator-product expansion. Finally, in sections 4 and 5 we will show that our axioms have much of the same power as the Wightman axioms by establishing "normal" (anti-)commutation relations and proving curved spacetime versions of the spin-statistics theorem and PCT theorem. Some further implications of our new perspective on quantum field theory are discussed in section 6 .

\section{General framework for the formulation of QFT}

We will now explain in much more detail our proposed framework for defining quantum field theory. We fix a dimension $D \geq 2$ of the spacetime and consider all $D$-dimensional globally hyperbolic spacetimes $\left(M, g_{a b}\right)$. As explained in the previous section, we will assume that each spacetime is equipped with an orientation, specified by a nowhere vanishing $D$-form $e_{a_{1} \ldots a_{D}}$ on $M$, and a time orientation, specified by (the equivalence class of) a globally defined time function $T: M \rightarrow \mathbb{R}$. The set of background data specified this way will be denoted

$$
\mathbf{M}=(M, g, T, e) .
$$

In certain cases, more background structure may be prescribed, such as a choice of bundles in which the quantum fields live. For example, if spinors are present and if $M$ admits more than one spin structure, then a choice of spin-structure over $M$ is assumed to be given as part of the background structure 2 , and is understood to also be part of M. It should be emphasized that two spacetimes with the same manifold and metric, but, e.g., with different time-orientations define distinct background structures. Below, we will consider quantum field theories associated with background structures, and we stress that, at this stage, the quantum field theories associated with different background structures (e.g., ones that merely differ in the choice of, say, time-orientation) need not have any relation whatsoever.

The quantum fields present in a given theory will be assumed to correspond to sections of vector bundles over $M$. We will denote the various quantum fields by $\phi^{(i)}$, with $i \in I$

\footnotetext{
${ }^{2}$ It is convenient to think of the background structure as a category [7], whose objects are the tuples M. Morphisms in the category of tuples $\mathbf{M}$ are isometric, causality, orientation, and other background structure preserving embeddings

$$
\rho: \mathbf{M} \rightarrow \mathbf{M}^{\prime} .
$$

Thus, $\rho$ is a diffeomorphism $M \rightarrow M^{\prime}$ such that $g=\rho^{*} g^{\prime}$, such that $\rho^{*} T^{\prime}$ represents the same time-orientation as $T$, such that $\rho^{*} e^{\prime}$ represents the same orientation as $e$, and such that the causal relations in $(M, g)$ inherited from $\left(M^{\prime}, g^{\prime}\right)$ coincide with the original ones. Furthermore, if $\mathbf{M}$ also includes the choice of a spin structure, then $\rho$ must also preserve the spin structures.
} 
where $I$ is a suitable indexing set, and we will write $V(i)$ for the vector bundle over $M$, of which $\phi^{(i)}$ corresponds to a section. It should be emphasized that $i \in I$ labels all of the quantum fields present in the theory, not just the "fundamental" ones. Thus, even if we were considering the theory of a single scalar field $\varphi$, there will be infinitely many composite fields of various tensorial types corresponding to all monomials in $\varphi$ and its derivatives, each of which would be labeled by a different index $i$. It will be convenient to also include a field denoted $\phi^{(\mathbf{1})}$ in the list of quantum fields, which will play the role of the identity element, $\mathbf{1}$, in the quantum field algebra.

We assume that each field $\phi^{(i)}$ has been assigned a Bose/Fermi parity $F(i)=0,1$ modulo two.

We further assume that there is an operation

$$
\star: I \rightarrow I \quad i \mapsto i^{\star},
$$

having the property that $V\left(i^{\star}\right)=\bar{V}(i)$, where for any vector space $E$, the vector space $\bar{E}$ consists of all anti-linear maps $E^{\mathrm{v}} \rightarrow \mathbb{C}$, with $E^{\mathrm{v}}$ denoting the dual space of $E$. In particular, if $i$ is associated with, say the vector bundle $V(i)$ of spinors with $P$ primed and $U$ unprimed indices, then $V\left(i^{\star}\right)$ is the bundle of spinors with $U$ primed and $P$ unprimed spinor indices. We demand that the star operation squares to the identity $i^{\star \star}=i$. We also require that $\phi^{\left(\mathbf{1}^{*}\right)}=\phi^{(\mathbf{1})}$, i.e. that $\mathbf{1}^{*}=\mathbf{1}$.

As in many other approaches to quantum field theory, we will use the smeared fields $\phi^{(i)}(f)$ - with $i \in I$, and $f$ a compactly supported test section in the dual vector bundle $V(i)^{\mathrm{v}}$ to $V(i)$ - to generate a ${ }^{*}$-algebra of observables, $\mathcal{A}(\mathbf{M})$. However, in most other algebraic approaches to quantum field theory, $\mathcal{A}(\mathbf{M})$ is assumed, a priori, to possess a particular topological and/or other structure (e.g., $\mathrm{C}^{*}$-algebra structure) and the algebraic relations within $\mathcal{A}(\mathbf{M})$ - together, perhaps, with specified actions of symmetry groups on $\mathcal{A}(\mathbf{M})$ - are assumed to encode all of the information about the quantum field theory under consideration. In particular, since the state space, $\mathcal{S}(\mathbf{M})$, is normally taken to consist of all positive linear maps on $\mathcal{A}(\mathbf{M})$, it is clear that $\mathcal{S}(\mathbf{M})$ cannot contain any information about the quantum field theory that is not already contained in $\mathcal{A}(\mathbf{M})$. We shall not proceed in this manner because it is far from clear to us what topological and other structure $\mathcal{A}(\mathbf{M})$ should be assumed to possess a priori in order to describe the quantum field theory. Instead, we shall view the theory as being specified by providing both an algebra of observables, $\mathcal{A}(\mathbf{M})$, and a space of allowed states, $\mathcal{S}(\mathbf{M})$. Essentially the only information about the theory contained in the algebra of obervables, $\mathcal{A}(\mathbf{M})$, will be the list of fields appearing in the theory and the relations that can be written as polynomial expressions in the fields and their derivatives. In our approach, the information normally encoded in the topology of $\mathcal{A}(\mathbf{M})$ will now be encoded in $\mathcal{S}(\mathbf{M})$. Of course, the semi-norms provided by $\mathcal{S}(\mathbf{M})$ could be used, a postiori, to define a topology on $\mathcal{A}(\mathbf{M})$, but it is not clear that this topology would encode all of the information in $\mathcal{S}(\mathbf{M})$; in any case, we find it simpler and more natural to consider the quantum field theory to be defined by the pair $\{\mathcal{A}(\mathbf{M}), \mathcal{S}(\mathbf{M})\}$.

The key idea of this paper is that we will obtain the pair $\{\mathcal{A}(\mathbf{M}), \mathcal{S}(\mathbf{M})\}$ in a natural (i.e., functorial) manner from the space of field labels $I$ and another datum, namely, the

\footnotetext{
${ }^{3}$ This operation gives $I$ the structure of an involutive category.
} 
collection of "operator product expansion (OPE) coefficients". The OPE coefficients are a family

$$
\mathcal{C}(\mathbf{M}) \equiv\left\{C_{(j)}^{\left(i_{1}\right) \cdots\left(i_{n}\right)}\left(x_{1}, \ldots, x_{n} ; y\right): \quad i_{1}, \ldots, i_{n}, j \in I, n \in \mathbb{N}\right\},
$$

where each $C_{(j)}^{\left(i_{1}\right) \cdots\left(i_{n}\right)}$ is a distribution on $M^{n+1}$, valued in the vector bundle

$$
E=V\left(i_{1}\right) \times \cdots \times V\left(i_{n}\right) \times V(j)^{\mathrm{v}} \stackrel{\pi}{\longrightarrow} M^{n+1}
$$

that is defined in some open neighborhood of the diagonal in $M^{n+1}$. Thus, given $\mathcal{C}(\mathbf{M})$, we will construct both the algebra $\mathcal{A}(\mathbf{M})$ and the state space $\mathcal{S}(\mathbf{M})$

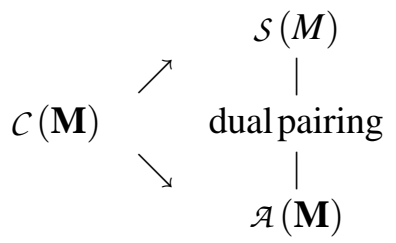

Thus, in our framework, a quantum field theory is uniquely specified by providing a list of quantum fields $I$ and a corresponding list of OPE coefficients $C(\mathbf{M})$. The OPE coefficients will be required to satisfy certain general properties, which, in effect, become the "axioms" of quantum field theory in curved spacetime. Most of the remainder of this section will be devoted to formulating these axioms. However, before providing the axioms for $\mathcal{C}(\mathbf{M})$, we briefly outline how the algebra $\mathcal{A}(\mathbf{M})$ and the state space $\mathcal{S}(\mathbf{M})$ are constructed from $\mathcal{C}(\mathbf{M})$ for any background structure $\mathbf{M}=(M, g, T, e)$.

The algebra $\mathcal{A}(\mathbf{M})$ is constructed by starting with the free algebra Free $(\mathbf{M})$ generated by all expressions of the form $\phi^{(i)}(f)$ with $i \in I$, and $f$ a compactly supported test section in the dual vector bundle to $V^{(i)}$. We define an antilinear *-operation on $\operatorname{Free}(\mathbf{M})$ by requiring that its action on the generators be given by

$$
\left[\phi^{(i)}(f)\right]^{*}=\phi^{\left(i^{\star}\right)}(\bar{f}),
$$

where $\bar{f} \in \operatorname{Sect}_{0}[\bar{V}(i)]$ is the conjugate test section to $f \in \operatorname{Sect}_{0}(V(i))$. The *-algebra $\mathcal{A}(\mathbf{M})$ is taken to be the resulting free $*$-algebra factored by a 2 -sided ideal generated by a set of polynomial relations in the fields and their derivatives. These relations consist of certain "universal" relations that do not depend on the particular theory under consideration (such as linearity of $\phi^{(i)}(f)$ in $f$ and (anti-)commutation relations) together with certain relations that may arise from the OPE coefficients $\mathcal{C}(\mathbf{M})$. A precise enumeration of the relations that define $\mathcal{A}(\mathbf{M})$ will be given at the beginning of section 3 .

The state space $\mathcal{S}(\mathbf{M})$ is a subspace of the space of all linear, functionals $\omega: \mathcal{A}(\mathbf{M}) \rightarrow \mathbb{C}$ that are positive in the sense that $\omega\left(A^{*} A\right) \equiv\left\langle A^{*} A\right\rangle_{\omega} \geq 0$ for all $A \in \mathcal{A}(\mathbf{M})$. This subspace is specified as follows: First, we require that for any state $\omega \in \mathcal{S}(\mathbf{M})$, the OPE coefficients in the collection $C(\mathbf{M})$ in eq. (6) appear in the expansion of the expectation value of the product of fields $\left\langle\phi^{\left(i_{1}\right)}\left(x_{1}\right) \cdots \phi^{\left(i_{n}\right)}\left(x_{n}\right)\right\rangle_{\omega}$ in terms of the fields $\left\langle\phi^{(j)}(y)\right\rangle_{\omega}$

$$
\left\langle\phi^{\left(i_{1}\right)}\left(x_{1}\right) \cdots \phi^{\left(i_{n}\right)}\left(x_{n}\right)\right\rangle_{\omega} \approx \sum_{j} C_{(j)}^{\left(i_{1}\right) \ldots\left(i_{n}\right)}\left(x_{1}, \ldots, x_{n} ; y\right)\left\langle\phi^{(j)}(y)\right\rangle_{\omega} .
$$


Here " $\approx$ " means that this equation holds in a suitably strong sense as an asymptotic relation in the limit that $x_{1}, \cdots, x_{n} \rightarrow y$. A precise definition of what is meant by this asymptotic relation will be given in eq. (39) below. Secondly, we require that $\omega$ satisfy a microlocal spectrum condition that, in essence, states that the singularities of $\left\langle\phi^{\left(i_{1}\right)}\left(x_{1}\right) \ldots \phi^{\left(i_{n}\right)}\left(x_{n}\right)\right\rangle_{\omega}$ are of "positive frequency type" in the cotangent space $T_{\left(x_{1}, \ldots, x_{n}\right)}^{*} M^{n}$. The precise form of this condition will be formulated in terms of the wave front set [22] (see eqs. (23) and (24) below).

We turn now to the formulation of the conditions that we shall impose on the OPE coefficients $C(\mathbf{M})$. As indicated above, in our framework, these conditions play the role of axioms for quantum field theory. Each operator product coefficient $C_{(j)}^{\left(i_{1}\right) \cdots\left(i_{n}\right)}$ in $C(\mathbf{M})$ is a distribution 4 on $M^{n+1}$, valued in the vector bundle $V\left(i_{1}\right) \times \cdots \times V\left(i_{n}\right) \times V(j)^{\mathrm{v}}$ that is defined in some open neighborhood of the diagonal in $M^{n+1}$. We will impose the following requirements on these coefficients:

C1) Locality and Covariance

C2) Identity element

C3) Compatibility with the $\star$-operation

C4) Commutativity/Anti-Commutativity

C5) Scaling Degree

C6) Asymptotic positivity

C7) Associativity

C8) Spectrum condition

C9) Analytic dependence upon the metric

Before formulating these conditions in detail, for each $i \in I$ we define the dimension, $\operatorname{dim}(i) \in \mathbb{R}$, of the field $\phi^{(i)}$ by 5

$$
\operatorname{dim}(i):=\frac{1}{2} \sup _{\text {backgrounds } \mathbf{M}} \operatorname{sd}\left\{C_{(\mathbf{1})}^{(i)\left(i^{\star}\right)}\right\}
$$

where "sd" denotes the scaling degree of a distribution (see appendix A) and it is understood that the scaling degree is taken about a point on the diagonal. In other words, $\operatorname{dim}(i)$

\footnotetext{
${ }^{4}$ More precisely, each OPE coefficient is an equivalence class of distributions, where two distributions are considered equivalent if their difference satisfies eq. (29) below for all $\delta>0$ and all T. Indeed, the OPE coefficients are more properly thought of as a sequence of (equivalence classes of) distributions, such that the difference between the $n$th and $m$ th terms in the sequence satisfies eq. (29) for $\delta=\min (m, n)$. However, to avoid such an extremely cumbersome formulation of our axioms and results, we will treat each OPE coefficient as a distribution.

${ }^{5}$ Note that, when $V(i)$ is not equal to $M \times \mathbb{C}$, i.e., when $\phi^{(i)}$ is not a scalar field, then quantities like $C_{(\mathbf{1})}^{(i)\left(i^{\star}\right)}$ are, by definition, distributions taking values in a vector bundle. What we mean by the scaling degree here and in similar equations in the following such as eq. (20) is the maximum of the scaling degrees of all "components" of such a bundle-valued distribution.
} 
measures the rate at which the coefficient of the identity 1 in the operator product expansion of $\phi^{(i)}\left(x_{1}\right) \phi^{\left(i^{\star}\right)}\left(x_{2}\right)$ blows up as $x_{1} \rightarrow x_{2}$. It will follow immediately from condition (C3) below that $\operatorname{dim}\left(i^{\star}\right)=\operatorname{dim}(i)$. Note also that $\operatorname{dim}(\mathbf{1})=0$.

For distributions $u_{1}$ and $u_{2}$ on $M^{n+1}$, we introduce the equivalence relation

$$
u_{1} \sim u_{2}
$$

to mean that the scaling degree of the distribution $u=u_{1}-u_{2}$ about any point on the total diagonal is $-\infty$. However, it should be noted that in the formulation of condition (C8) below and in the precise definition of the operator product expansion, eq. (39), we will need to consider limits where different points approach the total diagonal at different rates. We will then introduce a stronger notion of equivalence that, in effect, requires a scaling degree of $-\infty$ under these possibly different rates of approach. It is this stronger notion that was meant in eq. (9) above.

(C1) Covariance: Let $\rho: \mathbf{M} \rightarrow \mathbf{M}^{\prime}$ be a causality preserving isometric embedding preserving the orientations, spin-structures, and all other background structure, i.e. $\rho$ is a morphism in the category of background structures. We postulate that for each member of the above collection (6), we have

$$
\left(\rho^{*} \times \cdots \rho^{*} \times \rho_{*}^{-1}\right) C_{(j)}^{\left(i_{1}\right) \ldots\left(i_{n}\right)}\left[\mathbf{M}^{\prime}\right] \sim C_{(j)}^{\left(i_{1}\right) \ldots\left(i_{n}\right)}[\mathbf{M}] .
$$

(C2) Identity element: We require that

$$
C_{(j)}^{\left(i_{1}\right) \ldots(\mathbf{1}) \ldots\left(i_{n}\right)}\left(x_{1}, \ldots, x_{n} ; y\right)=C_{(j)}^{\left(i_{1}\right) \ldots\left(i_{k}\right)\left(i_{k+1}\right) \ldots\left(i_{n}\right)}\left(x_{1}, \ldots x_{k-1}, x_{k+1}, \ldots x_{n} ; y\right),
$$

where the identity $\mathbf{1}$ is in the $k$-th place. We also impose the following additional conditions on $C_{(j)}^{(i)}(x ; y)$, since these coefficients should merely implement a Taylor expansion of the operator with label $i$ localized at $x$ in terms of operators with labels $j$ localized at point $y$. As in a Taylor series, we demand that these coefficients depend only polynomially on the Riemannian normal coordinates of $x$ relative to $y$ (and are thus in particular smooth), and that

$$
C_{(j)}^{(i)}(x ; x)=\delta_{(j)}^{(i)} \operatorname{id}_{(i)}(x),
$$

where $\operatorname{id}_{(i)}(x)$ is the identity map in the fiber over $x$ of the vector bundle $V(i)$. Since a Taylor expansion of an operator at $x$ around another point $y$ involves the derivatives of the operators considered at $y$, and because derivatives tend to increase the dimension, we further demand that $C_{(j)}^{(i)}=0$ if $\operatorname{dim}(j)<\operatorname{dim}(i)$. Finally, if we Taylor expand a quantity at $x_{1}$ successively around a second point $x_{2}$, and then a third point $x_{3}$, this should be equivalent to expanding it in one stroke around the third point. Thus, we require that we have

$$
C_{(j)}^{(i)}\left(x_{1} ; x_{3}\right)=\sum_{(k)} C_{(k)}^{(i)}\left(x_{1} ; x_{2}\right) C_{(j)}^{(k)}\left(x_{2} ; x_{3}\right),
$$

where we note that the sum is only over the (finitely many) field labels $k$ such that $\operatorname{dim}(k) \leq$ $\operatorname{dim}(j)$. 
(C3) Compatibility with $\star$ : This relation encodes the fact that the underlying theory will have an operation analogous to the hermitian adjoint of a linear operator. The requirement is

$$
\overline{C_{(j)}^{\left(i_{1}\right) \ldots\left(i_{n}\right)}\left(x_{1}, \ldots, x_{n}, y\right)} \sim C_{\left(j^{\star}\right)}^{\left(i_{n}^{\star}\right) \ldots\left(i_{1}^{\star}\right)} \circ \pi\left(x_{1}, \ldots, x_{n}, y\right),
$$

where $\pi$ is the permutation

$$
\pi=\left(\begin{array}{ccccc}
1 & 2 & \ldots & n & n+1 \\
n & n-1 & \ldots & 1 & n+1
\end{array}\right)
$$

(C4) Commutativity/Anti-Commutativity: Let $\sigma$ be the permutation

$$
\sigma=\left(\begin{array}{cccccc}
1 & \ldots & k & k+1 & \ldots & n+1 \\
1 & \ldots & k+1 & k & \ldots & n+1
\end{array}\right)
$$

and let $F(i)$ be the Bose/Fermi parity of $\phi^{(i)}$. Then we have

$$
C_{(j)}^{\left(\sigma i_{1}\right) \ldots\left(\sigma i_{n}\right)} \circ \sigma\left(x_{1}, \ldots, x_{n}, y\right)=-(-1)^{F\left(i_{k}\right) F\left(i_{k+1}\right)} C_{(j)}^{\left(i_{1}\right) \ldots\left(i_{n}\right)}\left(x_{1}, \ldots, x_{n}, y\right)
$$

whenever $x_{k}$ and $x_{k+1}$ are spacelike separated (and in the neighborhood of the diagonal in $M^{n+1}$ where the OPE coefficients are actually defined).

(C5) Scaling Degree: We require that

$$
\operatorname{sd}\left\{C_{(k)}^{(i)(j)}\right\} \leq \operatorname{dim}(i)+\operatorname{dim}(j)-\operatorname{dim}(k) .
$$

(C6) Asymptotic Positivity: Let $i \in I$ be any given index. Then, for $D \geq 3$ we postulate that $\operatorname{dim}(i) \geq 0$, and that $\operatorname{dim}(i)=0$ if and only if $i=\mathbf{1}$. Note that, because we are taking the supremum over all spacetimes in eq. (10), our requirement that $\operatorname{dim}(i)>0$ for $i \neq \mathbf{1}$ does not imply that the scaling degree of $C_{(\mathbf{1})}^{\left.(i) i^{\star}\right)}$ for $i \neq \mathbf{1}$ is positive for all spacetimes, since the coefficient may e.g. "accidentally" happen to have a lower scaling degree for certain spacetimes of high symmetry, as happens for certain supersymmetric theories on Minkowski spacetime.

On a spacetime $M$ where the scaling degree of $C_{(\mathbf{1})}^{(i)\left(i^{\star}\right)}$ is $\operatorname{dim}(i)$, we know that if we scale the arguments of this distribution together by a factor of $\lambda$, and multiply by a power of $\lambda$ less than $2 \operatorname{dim}(i)$, then the resulting family of distributions cannot be bounded as $\lambda \rightarrow 0$. For our applications below, it is convenient to have a slightly stronger property, which we now explain. Let $X^{a}$ be a vector field on $M$ locally defined near $y$ such that $\nabla_{a} X^{b}=-\delta_{a}{ }^{b}$ at $y$. Let $\Phi_{t}$ be the flow of this field, which scales points by a factor of $e^{-t}$ relative to $y$ along the flow lines of $X^{a}$. If $f$ is a compactly supported test section in $V(i)$, we set $f_{\lambda}=\lambda^{-D} \Phi_{\log \lambda}^{*} f$. 
This family of test sections becomes more and more sharply peaked at $y$ as $\lambda \rightarrow 0$. We postulate that, for any $\delta>0$ and any $X^{a}$ as above, there exists an $f$ such that

$$
\lim _{\lambda \rightarrow 0}\left|\lambda^{2 \operatorname{dim}(i)-\delta} \int_{M \times M} C_{(\mathbf{1})}^{(i)\left(i^{\star}\right)}\left(x_{1}, x_{2}, y\right) f_{\lambda}\left(x_{1}\right) \bar{f}_{\lambda}\left(x_{2}\right) d \mu_{1} d \mu_{2}\right|=\infty,
$$

uniformly in $y$ in some neighborhood. This statement is slightly stronger than the statement that the scaling degree of our distribution is $\operatorname{dim}(i)$ on $M$, since the latter would only imply that the rescaled distributions under the limit sign in eq. (21) contain a subsequence that is unbounded in $\lambda$ for some test section $F\left(x_{1}, x_{2}\right)$ in $V(i) \times V\left(i^{\star}\right)$, not necessarily of the form $f\left(x_{1}\right) \bar{f}\left(x_{2}\right)$.

The reason for the terminology "asymptotic positivity axiom" arises from lemma 2 below. An alternative essentially equivalent formulation of this condition, which is related to "quantum inequalities", is given in Appendix B.

In $D=2$ spacetime dimensions, the above form of the asymptotic positivity condition is in general too restrictive. The reason is that in $D=2$, there are usually many fields $\phi^{(i)}$ of dimension $\operatorname{dim}(i)=0$ different from the identity operator. For example, for a free Klein-Gordon field, the basic field $\varphi$ and all its Wick-powers have vanishing dimensionin fact, their OPE-coefficients have a logarithmic scaling behavior. A possible way to deal with this example would be to consider only composite fields containing derivatives, as this subspace of fields is closed under the OPE. For this subspace of fields, the asymptotic positivity condition would then hold as stated. Another possibility is to introduce a suitably refined measure of the degree of divergence of the OPE coefficients also taking into account logarithms. Such a concept would clearly be sensible for free or conformal field theories in $D=2$, and it would also be adequate in perturbation theory (to arbitrary but finite orders). A suitable refinement of the above asymptotic positivity condition could then be defined, and all proofs given in the remainder of this paper would presumably still hold true, with minor modifications. For simplicity, however, we will not discuss this issue further in this paper, and we will stick with the asymptotic positivity condition in the above form.

(C7) Spectrum condition: The spectrum condition roughly says that the singularities of a field product ought to be of "positive frequency type," and is completely analogous the condition imposed on states that we will impose below: We demand that, near the diagonal, the wave front set (see Appendix $\mathrm{A}$ ) of the OPE coefficient satisfies

$$
\mathrm{WF}\left(C_{(j)}^{\left(i_{1}\right) \ldots\left(i_{n}\right)}\right) \subset \Gamma_{n}(\mathbf{M}) \times Z^{*} M
$$

where the last factor $Z^{*} M$ is the zero section of $T^{*} M$ and corresponds to the reference point $y$ in the OPE, and where the set $\Gamma_{n}(\mathbf{M}) \subset T^{*} M^{n} \backslash\{0\}$ is defined as follows. Consider embedded graphs $G(\vec{x}, \vec{y}, \vec{p}) \in \mathcal{G}_{m, n}$ in the spacetime manifold $M$ which have the following properties. Each graph $G$ has $n$ so-called "external vertices", $x_{1}, \ldots, x_{n} \in M$, and $m$ socalled "internal" or "interaction vertices" $y_{1}, \ldots, y_{m} \in M$. These vertices are of arbitrary valence, and are joined by edges, $e$, which are null-geodesic curves $\gamma_{e}:(0,1) \rightarrow M$. It is assumed that an ordering of the vertices is defined, and that the ordering among the 
external vertices is $x_{1}<\cdots<x_{n}$, while the ordering of the remaining interaction vertices is unconstrained. If $e$ is an edge joining two vertices, then $s(e)$ (the source) and $t(e)$ (the target) are the two vertices $\gamma_{e}(0)$ and $\gamma_{e}(1)$, where the curve is oriented in such a way that it starts at the smaller vertex relative to the fixed vertex ordering. Each edge carries a future directed, tangent parallel covector field, $p_{e}$, meaning that $\nabla_{\dot{\gamma}_{e}} p_{e}=0$, and $p_{e} \in \partial V^{+}$. With this notation set up, we define

$$
\begin{aligned}
\Gamma_{n, m}(M, g)= & \left\{\left(x_{1}, k_{1} ; \ldots ; x_{n}, k_{n}\right) \in T^{*} M^{n} \backslash\{0\} \mid \exists \text { decorated graph } G(\vec{x}, \vec{y}, \vec{p}) \in \mathcal{G}_{m, n}\right. \\
& \text { such that } y_{i} \in J^{+}\left(\left\{x_{1}, \ldots, x_{n}\right\}\right) \cap J^{-}\left(\left\{x_{1}, \ldots, x_{n}\right\}\right) \text { for all } 1 \leq i \leq m, \\
& \text { such that } k_{i}=\sum_{e: s(e)=x_{i}} p_{e}-\sum_{e: t(e)=x_{i}} p_{e} \text { for all } x_{i} \text { and } \\
& \text { such that } \left.0=\sum_{e: s(e)=y_{i}} p_{e}-\sum_{e: t(e)=y_{i}} p_{e} \text { for all } y_{i}\right\}
\end{aligned}
$$

where $J^{ \pm}(U)$ is the causal future resp. past of a set $U \subset M$, defined as the set of points that can be reached from $U$ via a future resp. past directed causal curve. We set

$$
\Gamma_{n}=\overline{\bigcup_{m \geq 0} \Gamma_{m, n}}
$$

Note in particular that the microlocal spectrum condition implies that the dependence of our OPE coefficients (6) on the reference point $y$ is smooth. When the spacetime is real analytic, we require a similar condition for the "analytic" wave front set [22] $\mathrm{WF}_{A}$ of the OPE coefficient.

Our formulation of the microlocal spectrum condition is a weaker condition than that previously proposed in [5], based on earlier work of [24]. The microlocal spectrum condition of [5] is satisfied by the correlation functions of suitable Hadamard states in linear field theory, but need not hold even perturbatively for interacting fields. In essence, our formulation allows for the presence of interaction vertices, thus weakening the condition relative to the free field case. Our condition can be shown to hold for perturbative interacting fields [15].

(C8) Associativity: Following [15], a notion of associativity is formulated by considering configurations $\left(x_{1}, \ldots, x_{n}\right)$ of points in $M^{n}$ (where $n>2$ ) approaching a point $y \in M$ at different rates. For example, if we have 3 points $\left(x_{1}, x_{2}, x_{3}\right)$, we may consider all points coming close to each other at the same rate, i.e., assume that their mutual distances are of order $\varepsilon$, where $\varepsilon \rightarrow 0$. Alternatively, we may consider a situation in which, say, $x_{1}, x_{2}$ approach each other very fast, say, at rate $\varepsilon^{2}$, while $x_{3}$ approaches $x_{1}, x_{2}$ at a slower rate, say at rate $\varepsilon$. The first situation corresponds, intuitively, to the process of performing the OPE of a triple product of operators "at once", while the second situation corresponds to first performing an OPE in the factors corresponding to $x_{1}, x_{2}$, and then successively performing a second OPE between the resulting fields and the third field situated at $x_{3}$. Obviously, for an 
arbitrary number $n$ of points, there are many different possibilities in which configurations can come close. We classify the different possibilities in terms of "merger trees," T. Each merger tree will give rise to a separate associativity condition.

For this, one constructs curves in $M^{n}$ parametrized by $\varepsilon$, which are in $M_{0}^{n}$ (the space $M^{n}$ minus all its diagonals) for $\varepsilon>0$, and which tend to a point on the diagonal as $\varepsilon \rightarrow 0$. These curves are labeled by trees $\mathbf{T}$ that characterize the subsequent mergers of the points in the configuration as $\varepsilon \rightarrow 0$. A convenient way to formally describe a tree $\mathbf{T}$ (or more generally, the disjoint union of trees, a "forest") is by a nested set $\mathbf{T}=\left\{S_{1}, \ldots, S_{k}\right\}$ of subsets $S_{i} \subset\{1, \ldots, n\}$. "Nested" means that two sets are either disjoint, or one is a proper subset of the other. We agree that the sets $\{1\}, \ldots,\{n\}$ are always contained in the tree (or forest). Each set $S_{i}$ in $\mathbf{T}$ represents a node of a tree, i.e., the set of vertices $\operatorname{Vert}(\mathbf{T})$ is given by the sets in $\mathbf{T}$, and $S_{i} \subset S_{j}$ means that the node corresponding to $S_{i}$ can be reached by moving downward from the node represented by $S_{j}$. The $\operatorname{root}(\mathrm{s})$ of the tree(s) correspond to the maximal elements, i.e., the sets that are not subsets of any other set. If the set $\{1, \ldots, n\} \in \mathbf{T}$, then there is in fact only one tree, while if there are several maximal elements, then there are several trees in the forest, each maximal element corresponding to the root of the respective tree. The leaves correspond to the sets $\{1\}, \ldots,\{n\}$, i.e., the minimal elements. For example for a configuration of $n=4$ points, a tree might look like in the following figure, and the corresponding nested set of subsets is also given.

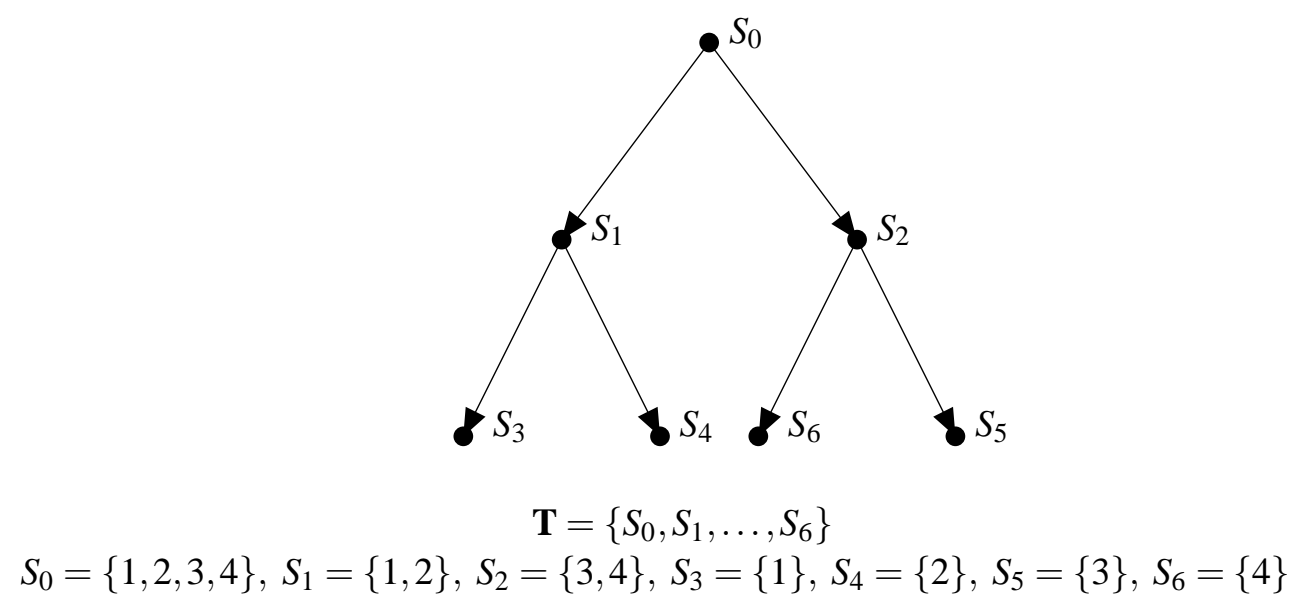

Figure 1 .

In the following, we will consider only $\mathbf{T}$ with a single root. The desired curves $\vec{x}(\varepsilon)$ tending to the diagonal are associated with $\mathbf{T}$ and are constructed as follows. First, we construct Riemannian normal coordinates around the reference point $y$, so that each point in a convex normal neighborhood of $y$ may be identified with a tangent vector $v \in T_{y} M$. We then choose a tetrad and further identify $T_{y} M \cong \mathbb{R}^{D}$, so that $v$ is in fact viewed as an element in $\mathbb{R}^{D}$. With each set $S \in \mathbf{T}$, we now associate a vector $v_{S} \in \mathbb{R}^{D}$, which we collect in a tuple

$$
\vec{v}=\left(v_{S_{1}}, \ldots, v_{S_{r}}\right) \in\left(\mathbb{R}^{D}\right)^{|\mathbf{T}|}, \quad \mathbf{T}=\left\{S_{1}, \ldots, S_{r}\right\},
$$

and we agree that $v_{\{1, \ldots, n\}}=0$, and where $|\mathbf{T}|$ is the number of nodes of the tree, i.e. the 
number of elements of the set $\mathbf{T}$. For $\varepsilon>0$, we define a mapping

$$
\psi_{\mathbf{T}}(\varepsilon):\left(\mathbb{R}^{D}\right)^{|\mathbf{T}|} \mapsto\left(\mathbb{R}^{D}\right)^{|\mathbf{T}|},\left(v_{S_{1}}, \ldots, v_{S_{r}}\right) \mapsto\left(x_{S_{1}}(\varepsilon), \ldots, x_{S_{r}}(\varepsilon)\right)
$$

by the formula

$$
x_{S}(\varepsilon)=\sum_{S^{\prime} \subset S} \varepsilon^{\operatorname{depth}\left(S^{\prime}\right)} v_{S^{\prime}}
$$

where $\operatorname{depth}\left(S^{\prime}\right)$ is defined as the number of nodes that connect $S^{\prime}$ with the root of the tree $\mathbf{T}$. For $\varepsilon$ sufficiently small, and $\vec{v}$ in a ball $B_{1}(0)^{|\mathbf{T}|}$, the vectors $x_{S}(\varepsilon) \in \mathbb{R}^{D}$ may be identified with points in $M$ via the exponential map. If the vectors $v_{S}$ satisfy the condition that, $v_{S^{\prime}} \neq v_{S^{\prime \prime}}$ for any $S^{\prime}, S^{\prime \prime}$ that are connected to a common $S$ by an edge, then the vector $\left(x_{\{1\}}(\varepsilon), \ldots, x_{\{n\}}(\varepsilon)\right) \in M^{n}$ does not lie on any of the diagonals, i.e., any pair of entries are distinct from each other. Its value as $\varepsilon \rightarrow 0$ approaches the diagonal of $M^{n}$. The $i$-th point in the configuration $x_{\{i\}}(\varepsilon)$ is obtained starting from $y$ by following the branches of the tree towards the $i$-th leaf, moving along the first edge by an amount $\varepsilon$ in the direction of the corresponding $v_{S}$ then the second by an amount $\varepsilon^{2}$ in the direction of the corresponding $v_{S}$, and so fourth, until the $i$-th leaf is reached. The curve $\left(x_{\{1\}}(\varepsilon), \ldots, x_{\{n\}}(\varepsilon)\right) \in M^{n}$ thus represents a configuration of points which merge hierarchically according to the structure of the tree $\mathbf{T}$, as $\varepsilon \rightarrow 0$. That is, the outermost branches of the tree merge at the highest order in $\varepsilon$, i.e., at rate $\varepsilon^{\text {depth ofbranch }}$, then the next level at a lower order, and so fourth, while the branches closest to the root merge at the slowest rate, $\varepsilon$. The following figure illustrates our definition.

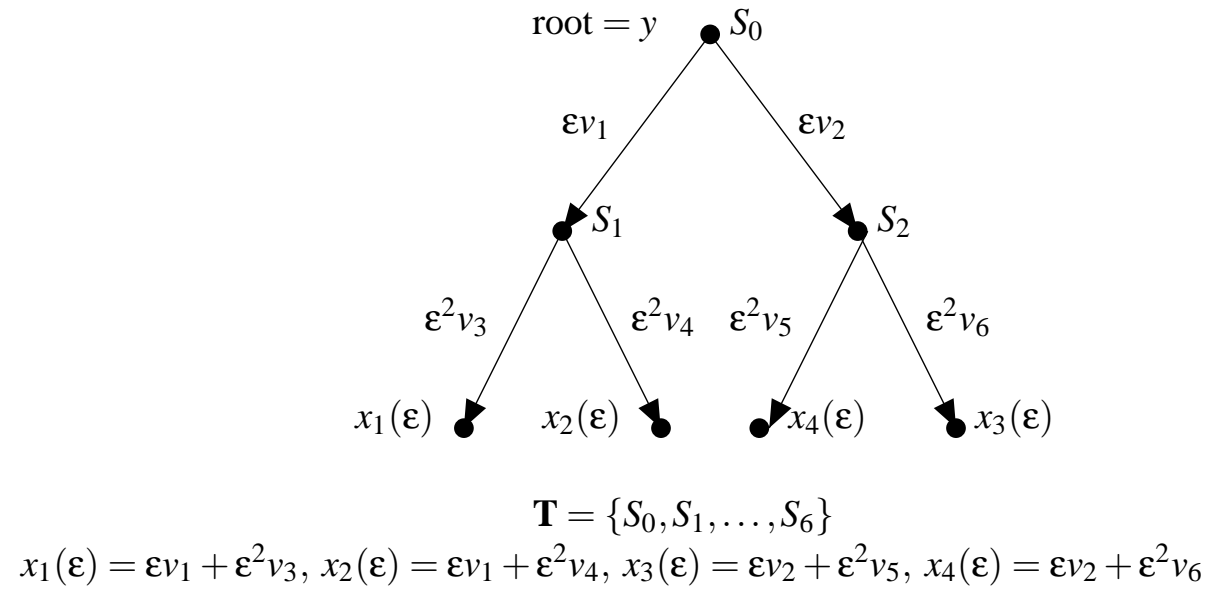

Figure 2

Thus, the points are scaled towards the diagonal of $M^{n}$, even though possibly at different speeds, and the limiting element as $\varepsilon \rightarrow 0$ is the element $(y, \ldots, y)$ on the diagonal 6 .

Using the maps $\psi_{\mathbf{T}}(\varepsilon)$ we can define an asymptotic equivalence relation $\sim_{\mathbf{T}, \delta}$ for distributions $u$ defined on $M^{|\mathbf{T}|}$. For points within a convex normal neighborhood, and sufficiently

\footnotetext{
${ }^{6}$ However, if the vector $\left(x_{\{1\}}(\varepsilon), \ldots, x_{\{n\}}(\varepsilon)\right) \in M^{n}$ is alternatively viewed as an element of the "FultonMacPherson compactification" $M_{c}^{n}$ of the configuration space $M^{n}$, then its limiting value may be viewed alternatively as lying in the boundary $\partial M_{c}^{n}$ of the compactification, and the vectors $\vec{v}$ may be viewed as defining a
} 
small $\varepsilon>0$, we can define the pull-back $u \circ \psi_{\mathbf{T}}(\varepsilon)$. This may be viewed a distribution in the variables $v_{S} \in \mathbb{R}^{D}, S \in \mathbf{T}$. We now define

$$
u \sim_{\mathbf{T}, \delta} 0 \quad: \Longleftrightarrow \lim _{\varepsilon \rightarrow 0+} \varepsilon^{-\delta} u \circ \psi_{\mathbf{T}}(\varepsilon)=0 \quad \text { for } \delta>0,
$$

in the sense of distributions defined on a neighborhood of the origin in $\left(\mathbb{R}^{D}\right)^{|\mathbf{T}|}$. We write $u \approx 0$ if $u \sim_{\mathbf{T}, \delta} 0$ for all $\mathbf{T}$ and all $\delta$. The condition that $u \approx 0$ is stronger than the previously defined condition $u \sim 0$ [see eq. (11)], which corresponds to the requirement that $u \sim_{\mathbf{T}, \delta} 0$ for all $\delta$ only for the trivial tree $\mathbf{T}=\{\{1\}, \ldots\{n\},\{1, \ldots, n\}\}$.

We can now state the requirement of associativity. Recall that if $\mathbf{T}$ is a tree with $n$ leaves, then $\psi_{\mathbf{T}}(\varepsilon)$ gives a curve in the configuration space of $n$ points in $M$ which representing the process of a subsequent hierarchical merger of the points according to the structure of the tree. If a subset of points in $\left(x_{\{1\}}(\varepsilon), \ldots, x_{\{n\}}(\varepsilon)\right)$ merges first, then one intuitively expects that one should be able to perform the OPE in those points first, and then subsequently perform OPE's of the other points in the hierarchical order represented by the tree. We will impose this as the associativity requirement. For example, if we have 4 points, and the tree corresponds to the nested set of subsets $\mathbf{T}=\{\{1,2\},\{3,4\},\{1,2,3,4\}\}$ as in the above figure, the pairs of points $x_{\{1\}}(\varepsilon), x_{\{2\}}(\varepsilon)$ respectively $x_{\{3\}}(\varepsilon), x_{\{4\}}(\varepsilon)$ approach each other at order $\varepsilon^{2}$, while the two groups then approach each other at a slower rate $\varepsilon$. We postulate that 7

$$
\begin{aligned}
& C_{\left(i_{5}\right)}^{\left(i_{1}\right)\left(i_{2}\right)\left(i_{3}\right)\left(i_{4}\right)}\left(x_{1}, x_{2}, x_{3}, x_{4} ; y\right) \sim \mathbf{T} \\
& \sum_{i_{6}, i_{7}} C_{\left(i_{6}\right)}^{\left(i_{1}\right)\left(i_{2}\right)}\left(x_{1}, x_{2} ; x_{6}\right) C_{\left(i_{7}\right)}^{\left(i_{3}\right)\left(i_{4}\right)}\left(x_{3}, x_{4} ; x_{7}\right) C_{\left(i_{5}\right)}^{\left(i_{6}\right)\left(i_{7}\right)}\left(x_{6}, x_{7} ; y\right) .
\end{aligned}
$$

For the same product of operators, consider alternatively the tree $\mathbf{T}^{\prime}=\{\{1,2,3\},\{1,2,3,4\}\}$. The corresponding associativity relation for the OPE coefficient is now

$$
C_{\left(i_{5}\right)}^{\left(i_{1}\right)\left(i_{2}\right)\left(i_{3}\right)\left(i_{4}\right)}\left(x_{1}, x_{2}, x_{3}, x_{4} ; y\right) \sim \mathbf{T}^{\prime} \sum_{i_{6}} C_{\left(i_{6}\right)}^{\left(i_{1}\right)\left(i_{2}\right)\left(i_{3}\right)}\left(x_{1}, x_{2}, x_{3} ; x_{6}\right) C_{\left(i_{5}\right)}^{\left(i_{6}\right)\left(i_{4}\right)}\left(x_{6}, x_{4} ; y\right)
$$

It is important to note, however, that there is in general no simple relation between the right hand sides of eqs. (30), (31) for different trees $\mathbf{T}$ and $\mathbf{T}^{\prime}$.

The corresponding relation for arbitrary numbers of points, and arbitrary types of trees is a straightforward generalization of this case, the only challenge being to introduce an appropriate notation to express the subsequent OPE's. For this, we consider maps $\vec{i}: \mathbf{T} \rightarrow I$ which associate with every node $S \in \mathbf{T}$ of the tree an element $i_{S} \in I$, the index set labelling

coordinate system of that boundary, which thereby has the structure of a stratifold

$$
\partial M_{c}^{n} \cong \bigcup_{\mathbf{T}} M[\mathbf{T}],
$$

with each face $\mathbf{T}$ corresponding to a lower dimensional subspace associated with a given merger tree [1].

${ }^{7}$ Here, the distribution on the left side is viewed as a distribution in $x_{1}, \ldots, x_{7}, y$ with a trivial dependence on $x_{5}, x_{6}, x_{7}$. 
the fields. If $S \in \mathbf{T}$, we let $S(1), S(2), \ldots S(r)$ be the branches of this tree, i.e. the nodes connected to $S$ by a single upward edge. With these notations in place, the generalization of eqs. (30) and (31) for an arbitrary number $n$ of points, and an arbitrary tree $\mathbf{T}$ is as follows. Let $\mathbf{T}$ be an arbitrary tree on $n$ elements, and let $\delta>0$ be an arbitrary real number. Then we have 8

$$
C_{(j)}^{\left(i_{1}\right) \ldots\left(i_{n}\right)}\left(x_{1}, \ldots, x_{n} ; y\right) \sim_{\mathbf{T}, \delta} \sum_{\vec{i} \in \operatorname{Map}(\mathbf{T}, I)}\left(\prod_{S \in \mathbf{T}} C_{\left(i_{S}\right)}^{\left(i_{S(1)}\right) \ldots\left(i_{S(r)}\right)}\left(x_{S(1)}, \ldots, x_{S(r)} ; x_{S}\right)\right),
$$

where the sums are over $\vec{i}$ with the properties that

$$
i_{\{1\}}=i_{1}, \ldots, i_{\{n\}}=i_{n}, \quad i_{\{1, \ldots, n\}}=j .
$$

The sum over $\vec{i}$ is finite, with $\operatorname{dim}\left(i_{S}\right) \leq \Delta$, where $\Delta$ is a number depending on the tree $\mathbf{T}$ and the real number $\delta$. Furthermore, it is understood that $x_{\{1\}}=x_{1}, \ldots, x_{\{n\}}=x_{n}$, and that $x_{\{1, \ldots, n\}}=y$.

(C9) Analytic and smooth dependence: Due to requirement (C1), the OPE coefficients may be regarded as functionals of the spacetime metric. We require that the distributions $C_{(j)}^{\left(i_{1}\right) \ldots\left(i_{n}\right)}$ have an analytic dependence upon the spacetime metric. For this, let $g^{(s)}$ be a 1-parameter family of analytic metrics, depending analytically on $s \in \mathbb{R}$. Then the corresponding OPE-coefficients $C_{(j)}^{\left(i_{1}\right) \ldots\left(i_{n}\right)}$ are distributions in $x_{1}, \ldots, x_{n}, y$ that also depend on the parameter $s$. We demand that the dependence on $s$ is "analytic". It is technically somewhat involved to define what one precisely means by this, because $C_{(j)}^{\left(i_{1}\right) \ldots\left(i_{n}\right)}$ itself is not analytic, but instead a distribution in the spacetime points. The appropriate way to make this definition rigorous was provided in [20, 16]. Similarly, if the spacetime is only smooth, we require a corresponding smooth variation of the OPE coefficients under smooth variations of the metric.

\section{Construction of the QFT from the OPE coefficients}

Now that we have stated in detail all the desired properties of the OPE coefficients, we are ready to give the precise definition of quantum field theory. A quantum field theory in curved spacetime associated with a collection of OPE coefficients satisfying the above properties is the pair $\{\mathcal{A}(\mathbf{M}), \mathcal{S}(\mathbf{M})\}$ consisting of a $*$-algebra $\mathcal{A}(\mathbf{M})$ and a space of states $\mathcal{S}(\mathbf{M})$ on $\mathcal{A}(\mathbf{M})$ that is canonically defined by the operator product coefficients, $\mathcal{C}(\mathbf{M})$, for any choice of the background spacetime structure $\mathbf{M}$. The algebra $\mathcal{A}(\mathbf{M})$ is defined as follows. To begin, let Free $(\mathbf{M})$ be the free *-algebra generated by all expressions of the form $\phi^{(i)}(f)$ with $i \in I$, and $f$ a compactly supported test section in the vector bundle $V(i)$ associated with the tensor or spinor character of $\phi^{(i)}$. The algebra $\mathcal{A}(\mathbf{M})$ is obtained by factoring Free $(\mathbf{M})$ by a set of relations, which are as follows.

\footnotetext{
${ }^{8}$ Here, the distribution on the left is regarded as a distribution in $x_{S} ; S \in \mathbf{T}$, with a trivial dependence on the $x_{S}$ with $S$ not equal to $\{1\}, \ldots,\{n\},\{1, \ldots, n\}$.
} 
A1) Identity: We have $\phi^{(1)}(f)=\int f d \mu \cdot \mathbf{1}$.

A2) Linearity: For any complex numbers $a_{1}, a_{2}$, any test sections $f_{1}, f_{2}$, and any field $\phi^{(i)}$, we have $\phi^{(i)}\left(a_{1} f_{1}+a_{2} f_{2}\right)=a_{1} \phi^{(i)}\left(f_{1}\right)+a_{2} \phi^{(i)}\left(f_{2}\right)$. The linearity condition might be viewed as saying that, informally,

$$
\phi^{(i)}(f)=\int_{M} \phi^{(i)}(x) f(x) d \mu
$$

is a pointlike field that averaged against a smooth weighting function. We shall often use the informal pointlike fields as a notational device, with the understanding that all identities are supposed to be valid after formally smearing with a test function.

A3) Star operation: For any field $\phi^{(i)}$, and any test section $f \in \operatorname{Sect}_{0}(V(i))$, let $\bar{f} \in$ $\operatorname{Sect}_{0}(\bar{V}(i))$, be the conjugate test section. Then we require that

$$
\left[\phi^{(i)}(f)\right]^{*}=\phi^{\left(i^{\star}\right)}(\bar{f}) .
$$

A4) Relations arising from the OPE: Let $K \subset I$ be a subset of the index set, and let $c_{(i)}, i \in K$ be scalar valued differential operators [i.e., differential operators taking a section $V(i)$ to a scalar function on $M$ ], such that

$$
\operatorname{sd}\left\{\sum_{i, j \in K}\left(\bar{c}_{\left(i^{\star}\right)} \otimes c_{(j)}\right) C_{(k)}^{\left(i^{\star}\right)(j)} v^{(k)}\right\}<0,
$$

for all $v^{(k)} \in V(k)$ and all $k \in I$. Then we impose the relation

$$
0=\sum_{i \in K} c_{(i)} \phi^{(i)}(f)
$$

for all $f \in C_{0}^{\infty}(M)$, where the differential operators act in the sense of distributions. This relation can be intuitively understood as follows. Let $O(f)$ be the smeared quantum field defined by the right side of eq. (37). If we consider the OPE of the quantity $\left\langle O\left(x_{1}\right) O\left(x_{2}\right)^{*}\right\rangle_{\omega}$ in some state, then the scaling degree requirement (36) implies that the limit of this quantity as $x_{1}, x_{2} \rightarrow y$ is equal to 0 . Heuristically, this implies that $O(y)$ is a well-defined element at a sharp point $y$, not just after smearing with a test function $f$ as in eq. (37). Since $\left\langle O(y) O(y)^{*}\right\rangle_{\omega}=0$ in all states, one would heuristically conclude that also $O(y)$ should be 0 as an algebra element. This is what our requirement states.

The above requirement serves to eliminate any redundancies in the field content arising e.g. from initially viewing, say, a field $\varphi$ and $\square \varphi$ as independent fields, or from initially specifying a set of linearly dependent fields. More nontrivially, this requirement should also serve to impose field equations in $\mathcal{A}(\mathbf{M})$. For example, in $\lambda \varphi^{4}$-theory, we expect that a field equation of the form $\square \varphi-m^{2} \varphi-\lambda \varphi^{3}=0$ should hold, where $\varphi^{3}$ is a composite 
field in the theory that should appear in the operator product expansion of three factors of $\varphi$. If such a field equation holds, then clearly $\square \varphi-m^{2} \varphi-\lambda \varphi^{3}$ should have a trivial OPE with itself, i.e., all OPE coefficients of the product of this operator with itself should have arbitrary low scaling degree. Thus, in this example, if we take $c_{(1)}=\square-m^{2}, c_{(2)}=-\lambda$, and $\phi^{(1)}=\varphi, \phi^{(2)}=\varphi^{3}$, then eq. (36) should hold. Our requirement effectively demands that field equations hold if and only if they are implied by the OPE condition eq. (36).

A5) (Anti-)commutation relations: Let $\phi^{\left(i_{1}\right)}$ and $\phi^{\left(i_{2}\right)}$ be fields, and let $f_{1}$ and $f_{2}$ be test sections corresponding to their respective spinor or tensor character, whose supports are assumed to be spacelike separated. Then the relation

$$
\phi^{\left(i_{1}\right)}\left(f_{1}\right) \phi^{\left(i_{2}\right)}\left(f_{2}\right)+(-1)^{F\left(i_{1}\right) F\left(i_{2}\right)} \phi^{\left(i_{2}\right)}\left(f_{2}\right) \phi^{\left(i_{1}\right)}\left(f_{1}\right)=0 .
$$

holds in $\mathcal{A}(\mathbf{M})$.

Having defined the algebra $\mathcal{A}(\mathbf{M})$, we next define the state space $\mathcal{S}(\mathbf{M})$ to consist of all those linear functionals $\langle.\rangle_{\omega}: \mathcal{A}(\mathbf{M}) \rightarrow \mathbb{C}$ with the following properties:

S1) Positivity: The functional should be of positive type, meaning that $\left\langle A^{*} A\right\rangle_{\omega} \geq 0$ for each $A \in \mathcal{A}(\mathbf{M})$. Physically, $\langle A\rangle_{\omega}$ is interpreted as the expectation value of the observable $A$ in $\omega$.

S2) OPE: The operator product expansion holds as an asymptotic relation. By this we mean more precisely the following. Let $\phi^{\left(i_{1}\right)}, \ldots, \phi^{\left(i_{n}\right)}$ be any collection of fields, let $\delta>$ 0 be arbitrary but fixed, and let $\mathbf{T}$ be any merger tree as described in the associativity condition. Let $\sim_{\delta, \mathbf{T}}$ be the associated asymptotic equality relation between distributions of $n$ spacetime points that are defined in a neighborhood of the diagonal which was defined in the associativity condition [see eq. [29]. Then we require that

$$
\left\langle\phi^{\left(i_{1}\right)}\left(x_{1}\right) \cdots \phi^{\left(i_{n}\right)}\left(x_{n}\right)\right\rangle_{\omega} \sim_{\delta, \mathbf{T}} \sum_{j} C_{(j)}^{\left(i_{1}\right) \ldots\left(i_{n}\right)}\left(x_{1}, \ldots, x_{n} ; y\right)\left\langle\phi^{(j)}(y)\right\rangle_{\omega},
$$

where the sum is carried out over all $j$ such that $d(j) \leq \Delta$, where $\Delta$ is a number depending upon the tree $\mathbf{T}$, and the specified accuracy, $\delta$.

S3) Spectrum condition: We have

$$
\mathrm{WF}\left(\left\langle\phi^{\left(i_{1}\right)}\left(x_{1}\right) \ldots \phi^{\left(i_{n}\right)}\left(x_{n}\right)\right\rangle_{\omega}\right) \subset \Gamma_{n}(\mathbf{M}),
$$

where the set $\Gamma_{n}(\mathbf{M}) \subset T^{*} M^{n} \backslash\{0\}$ was defined above in eq. (23). 
As part of our definition of a quantum field theory, we make the final requirement that there is at least on state, i.e.,

$$
\mathcal{S}(\mathbf{M}) \neq \emptyset \quad \text { for all } \mathbf{M} \text {. }
$$

If the state space were empty, then this is a sign that the OPE is inconsistent, and does not define a physically acceptable quantum field theory.

Remarks: (1) The OPE coefficients enter the construction of the algebra $\mathcal{A}(\mathbf{M})$ only via condition (A4). However, they provide a strong restriction on the state space $\mathcal{S}(\mathbf{M})$ via condition (S2).

(2) If $\omega \in \mathcal{S}(\mathbf{M})$ and $A \in \mathcal{A}(\mathbf{M})$, then $\omega\left(A^{*} \cdot A\right)$ is a positive linear functional on $\mathcal{A}(\mathbf{M})$ [i.e., satisfying (S1)] which can also be shown to satisfy (S2). This functional can be identified with a vector state in the Hilbert space representation of $\mathcal{A}(\mathbf{M})$ obtained by applying the GNS construction to $\omega$, and is therefore in the domain of all smeared field operators. It is natural to expect that, in a reasonable quantum field theory, the OPE [i.e. (S3)] should hold in such states as well, but this does not appear to follow straightforwardly within our axiomatic setting.

(3) There are some apparent redundancies in our assumptions in that commutativity/anticommutativity conditions have been imposed separately on the OPE coefficients and the algebra (see conditions (C4) and (A5)), and microlocal spectrum conditions have been imposed separately on the OPE coefficients and the states (see conditions (C8) and (S3)). It is possible that our assumptions could be reformulated in such a way as to eliminate these redundancies, e.g., it is possible that condition (A5) might follow from condition (C4) with perhaps somewhat stronger assumptions about states. However, we shall not pursue these possibilities here.

The construction of the pair $\{\mathcal{A}(\mathbf{M}), \mathcal{S}(\mathbf{M})\}$ obviously depends only upon the data entering that construction, namely the set of all operator product coefficients $C(\mathbf{M})$, as well as the assignments $i \mapsto V(i)$ and $i \mapsto F(i)$ of the index set enumerating the fields with tensor/spinor character, and with Bose/Fermi character. Thus, any transformation on field space preserving the OPE and the Bose/Fermi character will evidently give rise to a corresponding isomorphism between the algebras, and a corresponding map between the state spaces. We now give a more formal statement of this obvious fact, and then point out some applications.

Let $L: I \rightarrow I, i \mapsto i^{\prime}=L i$ be a map with the following properties. (a) $L \mathbf{1}=\mathbf{1}$ (b) $L$ preserves the $\star$-operation on $I$, (c) $L$ preserves the assignment of Bose/Fermi character, i.e., $F\left(i^{\prime}\right)=F(i)$. Furthermore, assume that there is an embedding map $\psi: M \rightarrow M^{\prime}$ (not necessarily an isometry at this stage), and for each index $i$, there is a bundle map $\Psi_{(i)}$ characterized by the following commutative diagram (where $i^{\prime}=L i$ )

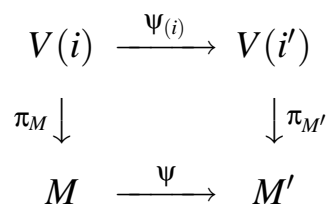

where $\pi_{M}$ respectively $\pi_{M^{\prime}}$ are the bundle projections associated with the vector bundles $V(i)$ and $V\left(i^{\prime}\right)$ over $M$ respectively $M^{\prime}$ that characterize the spinor/tensor character of the 
field labelled by $(i)$. Recalling that $V\left(i^{\star}\right)$ is required to be equal to the hermitian conjugate bundle $\bar{V}(i)$, and denoting by conj the operation of conjugation mapping between these bundles, we require as a consistency condition that

$$
\operatorname{conj}_{\mathbf{M}^{\prime}} \circ \psi_{(i)}=\Psi_{(i)^{\star}} \circ \operatorname{conj}_{\mathbf{M}},
$$

for all indices $i \in I$. We say that a collection of OPE coefficients $\mathcal{C}(\mathbf{M})$ on $\mathbf{M}=(M, g, T, e)$ and a collection $C^{\prime}\left(\mathbf{M}^{\prime}\right)$ on $\mathbf{M}^{\prime}=\left(M^{\prime}, g^{\prime}, T^{\prime}, e^{\prime}\right)$ are equivalent if

$$
\left(\psi_{\left(i_{1}\right)}^{*} \times \cdots \times \psi_{\left(i_{n}\right)}^{*} \times \psi_{(j) *}^{-1}\right) C^{\prime\left(L i_{1}\right) \ldots\left(L i_{n}\right)}\left[\mathbf{M}^{\prime}\right] \sim C_{(j)}^{\left(i_{1}\right) \ldots\left(i_{n}\right)}[\mathbf{M}]
$$

see eq. (11). By simply going through the definitions of the algebra $\mathcal{A}(\mathbf{M})$ and the state space $\mathcal{S}(\mathbf{M})$ it is then clear that the following (almost trivial) lemma holds.

Lemma 1. Under the consistency conditions (43) and (44), and assuming that $\psi$ preserves all background structure (i.e., $\psi$ is an isometric embedding preserving the causality relations, orientations, and spin structures) the map $\alpha_{L}: \mathcal{A}(\mathbf{M}) \mapsto \mathcal{A}\left(\mathbf{M}^{\prime}\right)$

$$
\alpha_{L}: \phi_{\mathbf{M}}^{(i)}(f) \mapsto \phi_{\mathbf{M}^{\prime}}^{\left(i^{\prime}\right)}\left(\psi_{(i) *} f\right), \quad i^{\prime}=L i
$$

defines a linear *-homomorphism. The dual map $\alpha_{L}^{\mathrm{v}}$ between the corresponding state spaces defines a map $\mathcal{S}\left(\mathbf{M}^{\prime}\right) \rightarrow \mathcal{S}(\mathbf{M})$.

Another way of stating this result is to view the maps $L$ as described above as morphisms in the category whose objects are the OPE-coefficient systems $\mathcal{C}(\mathbf{M})$. The above lemma then says that the constructions of $\mathcal{S}(\mathbf{M})$ and of $\mathcal{A}(\mathbf{M})$ from $\mathcal{C}(\mathbf{M})$ are functorial in nature.

We now discuss some applications of the lemma:

Application 1: Consider the case where $L=i d, \psi: M \rightarrow M^{\prime}$ is an isometric embedding preserving orientations and any other background structure, and $\psi_{(i)}$ is the natural bundle map associated with $\psi$. Then (43) obviously holds, while (44) holds because of the locality and covariance property of the OPE coefficients. The map $\alpha_{L}$, whose existence is guaranteed by the lemma, then corresponds to the map $\chi_{\rho}$ discussed in the introduction. In particular, the assignment

$$
\text { Background Structures } \rightarrow \text { Algebras }, \quad \mathbf{M} \rightarrow \mathcal{A}(\mathbf{M})
$$

is functorial, in the sense that if $\rho$ is an arrow in the category of background structuresi.e., an orientation and causality preserving isometric embedding from one spacetime into another-then $\chi_{\rho}$ is the corresponding arrow in the category of *-algebras-i.e., an injective *-homomorphism. Functoriality means that the assignment

$$
\text { Isometric Embeddings } \rightarrow \text { Algebra Homomorphisms }, \quad \rho \rightarrow \chi_{\rho}
$$

respects composition of arrows in the respective categories. Thus, in the terminology of the introduction, the assumptions of our framework define a local, covariant quantum field theory, and all fields $\phi^{(i)}$ are local, covariant quantum fields. Furthermore, $\mathcal{A}: \mathbf{M} \rightarrow \mathcal{A}(\mathbf{M})$ is a functor in the sense of [7]. 
Application 2: As the second application, consider a purely "internal" symmetry, $L$, i.e., consider the case that $\mathbf{M}=\mathbf{M}^{\prime}, \psi=i d$. Then $\alpha_{L}$ acts upon $\mathcal{A}(\mathbf{M})$ as a $*$-autmorphism, i.e., an internal symmetry. More generally, there could be an entire group $G$ of $\operatorname{such} L$ with the corresponding maps $\psi_{(i)}$ satisfying the composition law of the group, i.e., if we have $L, L^{\prime}$, then the map associated with $L^{\prime \prime}=L \circ L^{\prime}$ and $\psi^{\prime \prime}=\psi \circ \psi^{\prime}$ is given by $\psi_{(i)}^{\prime \prime}=\psi_{(i)} \circ \psi_{(i)}^{\prime}$. In this case, we get an action of $G$ on $\mathcal{A}(\mathbf{M})$ by $*$-automorphisms $\alpha_{L}$ satisfying the composition law $\alpha_{L} \circ \alpha_{L^{\prime}}=\alpha_{L^{\prime \prime}}$.

Another simple consequence of our axioms is the following lemma. As above in (C6), let $X^{a}$ be a vector field on $M$ locally defined near $y$ such that $\nabla_{a} X^{b}=-\delta_{a}{ }^{b}$ at $y$. Let $\Phi_{t}$ be the flow of this field 9 , which scales points relative to $y$ by a factor of $e^{-t}$. If $f$ is a compactly supported test section in $V(i)$, we set $f_{\lambda}=\lambda^{-D} \Phi_{\log \lambda}^{*} f$ for $\lambda>0$.

Lemma 2. For $i \neq \mathbf{1}$, there exists an $\mathbf{M}, \delta>0$, and test section $f$ such that

$$
\lim _{\lambda \rightarrow 0} \lambda^{2 \operatorname{dim}(i)-\delta} \int_{M \times M} C_{(\mathbf{1})}^{(i)\left(i^{\star}\right)}\left(x_{1}, x_{2}, y\right) f_{\lambda}\left(x_{1}\right) \bar{f}_{\lambda}\left(x_{2}\right) d \mu_{1} d \mu_{2}=+\infty .
$$

Proof: Let $\omega$ be an arbitrary state. Then we have $\left\langle\phi^{(i)}\left(f_{\lambda}\right) \phi^{\left(i^{\star}\right)}\left(\bar{f}_{\lambda}\right)\right\rangle_{\omega} \geq 0$, from the star axiom (C3), and the positivity of any state. Let $\mathbf{M}$ be such that the scaling degree of $C_{(\mathbf{1})}^{(i)\left(i^{\star}\right)}$ equals $2 \operatorname{dim}(i)$. By the scaling degree and asymptotic positivity axioms (C5) and (C6), for

sufficiently small $\delta>0$, the quantity $2 \operatorname{dim}(i)-\delta$ is bigger than the scaling degree of $C_{(j)}^{(i)\left(i^{\star}\right)}$ for any $j \neq \mathbf{1}$. Hence using eq. (39) and $\langle\mathbf{1}\rangle_{\omega}=1$, we have

$$
\lim _{\lambda \rightarrow 0} \lambda^{2 \operatorname{dim}(i)-\delta}\left\{\left\langle\phi^{(i)}\left(f_{\lambda}\right) \phi^{\left(i^{\star}\right)}\left(\bar{f}_{\lambda}\right)\right\rangle_{\omega}-C_{(\mathbf{1})}^{(i)\left(i^{\star}\right)}\left(f_{\lambda}, \bar{f}_{\lambda}\right)\right\}=0 .
$$

By axiom (C6), we can choose $f$ so that the second term tends to $\infty$ in absolute value as $\lambda \rightarrow$ 0 . However, the first term is always non-negative. Therefore, $C_{(\mathbf{1})}^{(i)\left(i^{\star}\right)}\left(f_{\lambda}, \bar{f}_{\lambda}\right) \rightarrow+\infty$.

\section{Normal (anti-) commutation relations}

In our axioms, we assumed that every field $\phi^{(i)}$ was either a Bose or Fermi field, i.e., that there was a consistent assignment $i \mapsto F(i) \in \mathbb{Z}_{2}$ such that (19) holds. A priori, there is no relation between $F(i)$ and the Bose/Fermi character of the corresponding hermitian conjugate field, i.e., $F\left(i^{\star}\right)$. We will now prove that, in fact, $F(i)=F\left(i^{\star}\right)$ as a consequence of our axioms.

Theorem 1. We have "normal (anti-)commutation relations," in the sense that

$$
F(i)=F\left(i^{\star}\right)
$$

for all $i \in I$.

\footnotetext{
${ }^{9}$ It follows that we can write $\Phi_{\log \lambda}(x)=y+\lambda^{-1}(x-y)$ in a suitable coordinate system covering $y$.
} 
Proof: Let $f$ and $h$ be compactly supported test sections with support in a convex normal neighborhood of a point $y \in M$. Let $\omega$ be a quantum state, i.e., a positive normalized linear functional $\mathcal{A}(\mathbf{M}) \rightarrow \mathbb{C}$. Using $(35)$, we see that positivity immediately implies that

$$
\left\langle\phi^{(i)}(f) \phi^{(i)}(h) \phi^{\left(i^{\star}\right)}(\bar{h}) \phi^{\left(i^{\star}\right)}(\bar{f})\right\rangle_{\omega} \geq 0 .
$$

Assume now that the supports of $f, h$ are spacelike separated. Then, using the (anti- )commutation relations eq. (38), it follows that

$$
p\left\langle\phi^{(i)}(f) \phi^{\left(i^{\star}\right)}(\bar{f}) \phi^{\left(i^{\star}\right)}(\bar{h}) \phi^{(i)}(h)\right\rangle_{\omega} \geq 0,
$$

where $p=(-1)^{F(i) F\left(i^{\star}\right)+F\left(i^{\star}\right)^{2}}$. Clearly, if we could show that the expectation value in this expression were positive for some test sections, $f, h$, in some spacetime, then it would follow that $p=+1$, i.e.

$$
F(i) F\left(i^{\star}\right)+F\left(i^{\star}\right)^{2}=0 \quad \bmod 2,
$$

and by reversing the roles of $i$ and $i^{\star}$, it would also follow that

$$
F(i) F\left(i^{\star}\right)+F(i)^{2}=0 \quad \bmod 2,
$$

from which the statement $F(i)=F\left(i^{\star}\right)$ modulo 2 would follow. Clearly, it suffices to show that the expectation value is asymptotically positive for a 1-parameter family of test sections $f_{\lambda}, h_{\lambda}$ whose supports are scaled towards $y \in M$ as $\lambda \rightarrow 0$.

To show this, we consider the particular merger tree $\mathbf{T}$ of figure 1, and the corresponding associativity condition. This tree corresponds to the scaling map $\Psi_{\mathbf{T}}(\varepsilon): \vec{x}(1) \rightarrow \vec{x}(\varepsilon)$, with

$$
\begin{aligned}
& x_{1}(\varepsilon)=\operatorname{Exp}_{y}\left(\varepsilon v_{1}+\varepsilon^{2} v_{3}\right) \\
& x_{2}(\varepsilon)=\operatorname{Exp}_{y}\left(\varepsilon v_{1}+\varepsilon^{2} v_{4}\right) \\
& x_{3}(\varepsilon)=\operatorname{Exp}_{y}\left(\varepsilon v_{2}+\varepsilon^{2} v_{5}\right) \\
& x_{4}(\varepsilon)=\operatorname{Exp}_{y}\left(\varepsilon v_{2}+\varepsilon^{2} v_{6}\right) \\
& x_{5}(\varepsilon)=\operatorname{Exp}_{y}\left(\varepsilon v_{1}\right) \\
& x_{6}(\varepsilon)=\operatorname{Exp}_{y}\left(\varepsilon v_{2}\right) \\
& x_{7}(\varepsilon)=y .
\end{aligned}
$$

The corresponding associativity condition together with (S2) yields

$$
\begin{aligned}
\lim _{\varepsilon \rightarrow 0} \varepsilon^{-\delta}\left(\left\langle\phi^{(i)}\left(x_{1}(\varepsilon)\right) \phi^{\left(i^{\star}\right)}\left(x_{2}(\varepsilon)\right) \phi^{\left(i^{\star}\right)}\left(x_{3}(\varepsilon)\right) \phi^{(i)}\left(x_{4}(\varepsilon)\right)\right\rangle_{\omega}\right. \\
-\sum_{j_{1}, j_{2}, j_{3}} C_{\left(j_{1}\right)}^{(i)\left(i^{\star}\right)}\left(x_{1}(\varepsilon), x_{2}(\varepsilon) ; x_{5}(\varepsilon)\right) C_{\left(j_{2}\right)}^{\left(i^{\star}\right)(i)}\left(x_{3}(\varepsilon), x_{4}(\varepsilon) ; x_{6}(\varepsilon)\right) \\
\left.\quad \times C_{\left(j_{3}\right)}^{\left(j_{1}\right)\left(j_{2}\right)}\left(x_{5}(\varepsilon), x_{6}(\varepsilon) ; y\right)\left\langle\phi^{\left(j_{3}\right)}(y)\right\rangle_{\omega}\right)=0 .
\end{aligned}
$$


This is to be understood in the sense of distributions in $v_{1}, \ldots, v_{6}$. The sums go over all indices with $\operatorname{dim}\left(j_{k}\right) \leq \Delta$, where $\Delta$ depends on $\delta$. We now use axioms (C5) and (C6) to analyze the scaling of the individual terms under the sum. It follows that

$$
\lim _{\varepsilon \rightarrow 0} \varepsilon^{\alpha} C_{\left(j_{1}\right)}^{(i)\left(i^{\star}\right)}\left(x_{1}(\varepsilon), x_{2}(\varepsilon) ; x_{5}(\varepsilon)\right) C_{\left(j_{2}\right)}^{\left(i^{\star}\right)(i)}\left(x_{3}(\varepsilon), x_{4}(\varepsilon) ; x_{6}(\varepsilon)\right) C_{\left(j_{3}\right)}^{\left(j_{1}\right)\left(j_{2}\right)}\left(x_{5}(\varepsilon), x_{6}(\varepsilon) ; y\right)=0
$$

if $\alpha>8 \operatorname{dim}(i)-\operatorname{dim}\left(j_{1}\right)-\operatorname{dim}\left(j_{2}\right)-\operatorname{dim}\left(j_{3}\right)$ in the sense of distributions. Thus, the term under the sum with the potentially most singular behavior as $\varepsilon \rightarrow 0$ is the one where $\operatorname{dim}\left(j_{1}\right)=\operatorname{dim}\left(j_{2}\right)=\operatorname{dim}\left(j_{3}\right)$ is minimal, i.e. equal to 0 , by axiom (C6). If these dimensions vanish, then by axiom (C6), we have $j_{k}=\mathbf{1}$.

Because $\langle\mathbf{1}\rangle_{\omega}=1$, and because the OPE coefficients involving only identity operators are equal to 1 by the identity axiom, we have

$$
\begin{aligned}
\lim _{\varepsilon \rightarrow 0} \varepsilon^{8 \operatorname{dim}(i)+\delta}\left(\left\langle\phi^{(i)}\left(x_{1}(\varepsilon)\right) \phi^{\left(i^{\star}\right)}\left(x_{2}(\varepsilon)\right) \phi^{\left(i^{\star}\right)}\left(x_{3}(\varepsilon)\right) \phi^{(i)}\left(x_{4}(\varepsilon)\right)\right\rangle_{\omega}\right. \\
\left.-C_{(\mathbf{1})}^{(i)\left(i^{\star}\right)}\left(x_{1}(\varepsilon), x_{2}(\varepsilon), x_{5}(\varepsilon)\right) C_{(\mathbf{1})}^{\left(i^{\star}\right)(i)}\left(x_{3}(\varepsilon), x_{4}(\varepsilon), x_{6}(\varepsilon)\right)\right)=0,
\end{aligned}
$$

for some $\delta>0$. We now integrate this expression against the test section $f\left(v_{3}\right) \bar{f}\left(v_{4}\right) \bar{h}\left(v_{5}\right) h\left(v_{6}\right)$, where $f, h$ are of compact support, and change integration variables 10 . Then we get for the terms under the limit sign

$$
\left\langle\phi^{(i)}\left(f_{\varepsilon}\right) \phi^{\left(i^{\star}\right)}\left(\bar{f}_{\mathcal{\varepsilon}}\right) \phi^{\left(i^{\star}\right)}\left(\bar{h}_{\varepsilon}\right) \phi^{(i)}\left(h_{\varepsilon}\right)\right\rangle_{\omega}-C_{(\mathbf{1})}^{(i)\left(i^{\star}\right)}\left(f_{\varepsilon}, \bar{f}_{\varepsilon}\right) C_{(\mathbf{1})}^{\left(i^{\star}\right)(i)}\left(\bar{h}_{\varepsilon}, h_{\varepsilon}\right)
$$

Here, we have defined $f_{\varepsilon}(x)=\varepsilon^{-2 D} f \circ \alpha_{1}(\varepsilon, x)$ and $h_{\varepsilon}(x)=\varepsilon^{-2 D} h \circ \alpha_{2}(\varepsilon, x)$ with $\alpha_{i}(\varepsilon,$.$) :$ $M \rightarrow \mathbb{R}^{D}$ are the maps that are defined in a sufficiently small neighborhood of $y$ by

$$
\begin{aligned}
& \alpha_{1}(\varepsilon, x)=\varepsilon^{-1} v_{1}+\varepsilon^{-2} \operatorname{Exp}_{y}^{-1}(x) \\
& \alpha_{2}(\varepsilon, x)=\varepsilon^{-1} v_{2}+\varepsilon^{-2} \operatorname{Exp}_{y}^{-1}(x) .
\end{aligned}
$$

Finally, we use the lemma 2 to conclude that there exist $f, h$ such that both $C_{(\mathbf{1})}^{(i)\left(i^{\star}\right)}\left(f_{\mathcal{\varepsilon}}, \bar{f}_{\mathcal{\varepsilon}}\right) \rightarrow$ $+\infty$ and $C_{(\mathbf{1})}^{(i)\left(i^{\star}\right)}\left(h_{\varepsilon}, \bar{h}_{\varepsilon}\right) \rightarrow+\infty$ for some spacetime and some subsequence of $\varepsilon \rightarrow 0$. In view of eq. (59), it follows that

$$
\lim _{\varepsilon \rightarrow 0}\left\langle\phi^{(i)}\left(f_{\varepsilon}\right) \phi^{\left(i^{\star}\right)}\left(\bar{f}_{\varepsilon}\right) \phi^{\left(i^{\star}\right)}\left(\bar{h}_{\varepsilon}\right) \phi^{(i)}\left(h_{\varepsilon}\right)\right\rangle_{\omega}=+\infty,
$$

so the expectation value (52) is positive for the choice of test sections $f, h$ given by $f_{\varepsilon}, h_{\varepsilon}$, see eq. (60), for sufficiently small $\varepsilon$. These test sections will have spacelike separated support as long as $\operatorname{Exp}_{y} v_{1}$ and $\operatorname{Exp}_{y} v_{2}$ are spacelike, which we may assume to be the case.

\footnotetext{
${ }^{10}$ We should also integrate against a test function in $v_{1}, v_{2}$. But the result 59) is already smooth in these variables, so we can omit this smearing.
} 


\section{The spin-statistics theorem and the PCT-theorem}

In this section, we prove that appropriate versions of the spin-statistics theorem and the PCT theorem hold in curved spacetime under our axiom scheme. We explicitly discuss the case when the spacetime dimension is even, $D=2 \mathrm{~m}$ and discuss the case of odd dimensions briefly in remark (2) below the PCT theorem. The key ingredient in both proofs is a relation, proven in in [16], between the OPE coefficients $C(\mathbf{M})$ on the background structure $\mathbf{M}=$ $(M, g, T, e)$, and the OPE coefficients $\mathcal{C}(\overline{\mathbf{M}})$ on the background structure

$$
\overline{\mathbf{M}}=(M, g,-T, e)
$$

consisting of the same manifold $M$, the same metric $g$, the same orientation $e$, but the opposite time orientation $T$. For even spacetime dimensions $D=2 m$, this relation is 11 :

$$
C_{(j)}^{\left(i_{1}\right) \ldots\left(i_{n}\right)}[\overline{\mathbf{M}}] \sim \overline{C_{\left(j^{\star}\right)}^{\left(i_{1}^{\star}\right) \ldots\left(i_{n}^{\star}\right)}[\mathbf{M}]} \cdot \begin{cases}\mathrm{i}^{-F(j)}(-1)^{-U(j)} \prod_{k=1}^{n} \mathrm{i}^{F\left(i_{k}\right)}(-1)^{U\left(i_{k}\right)} & m \text { even }, \\ \mathrm{i}^{-F(j)+U(j)-P(j)} \prod_{k=1}^{n} \mathrm{i}^{F\left(i_{k}\right)-U\left(i_{k}\right)+P\left(i_{k}\right)} & m \text { odd },\end{cases}
$$

where $\mathrm{i}=\sqrt{-1}$. Here, we recall that with each quantum field $\phi^{(i)}$ there is associated a bundle $V(i)$ over $M$ corresponding to the tensor or spinor character of the quantum field. In even spacetime dimensions $D=2 m$, such a bundle $V(i)$ is a tensor product

$$
V(i)=S_{-}^{\otimes U(i)} \otimes S_{+}^{\otimes P(i)},
$$

where the first factor corresponds to the $U(i)$ "unprimed-" and the second to the $P(i)$ "primed" spinor indices. More precisely, the bundles $S_{ \pm}$are defined as the \pm 1 eigenspaces of the chirality operator 12

$$
\Gamma=\frac{1}{D !}(-1)^{(m-1)(2 m-1) / 2} e^{a_{1} a_{2} \ldots a_{D}} \gamma_{a_{1}} \gamma_{a_{2}} \cdots \gamma_{a_{D}}, \quad \Gamma^{2}=i d_{S},
$$

acting on a $2^{m}$-dimensional complex vector bundle $S$ over $M$ of "Dirac spinors". This bundle $S$ corresponds to a fundamental representation of the Clifford algebra (in the tangent bundle) generated by the curved space gamma-matrices $\gamma_{a}$. There exists a linear isomorphism $c: S \rightarrow \bar{S}$, where $\bar{S}$ is the conjugate bundle of anti-linear maps $S^{\mathrm{v}} \rightarrow \mathbb{C}$. Owing to the relation $\bar{\Gamma}=(-1)^{m-1} c \Gamma c^{-1}$, it follows that for even $m$, the bundles $S_{ \pm}$and $\bar{S}_{\mp}$ are isomorphic via $c$, while for odd $m$ the bundles $S_{ \pm}$and $\bar{S}_{ \pm}$are isomorphic, and we will hence always identify these bundles. Thus, for even $m$ the roles of primed and unprimed spinor indices [i.e., respective tensor factors in eq. (65)] are exchanged when passing to the hermitian adjoint $\phi^{\left(i^{\star}\right)}$ of a quantum field $\phi^{(i)}$, while for odd $m$ the roles are not exchanged.

We also note that the coefficients on the right side of eq. (64) are sections in the (tensor product of the) spin bundles $V_{\mathbf{M}}(i)$ referring to the time function $T$ associated with $\mathbf{M}$, while the coefficients on the left side are sections in the spinor bundles $V_{\overline{\mathbf{M}}}\left(i^{\star}\right)$ defined via

\footnotetext{
${ }^{11}$ Note that the "bar" symbol is referring to the $P T$-reversed background structure in the term on the left side, while it means hermitian conjugation on the right side.

${ }^{12}$ Here, the orientation $D$-form is normalized so that $g^{a_{1} b_{1}} \ldots g^{a_{D} b_{D}} e_{a_{1} \ldots a_{D}} e_{b_{1} \ldots b_{D}}=-D$ !.
} 
the opposite time orientation $-T$ associated with $\overline{\mathbf{M}}$. As explained in [16], there is a natural identification map between these bundles, and this identification map is understood in (64).

The proof of (64) makes use of the microlocal, analytical, and causal properties of the OPE coefficients and proceeds via analytic continuation [16]. Since it is the main input in the proofs of both the spin-statistics theorem and the PCT theorem, we now outline, following [16], how (64) is proven within our axiomatic setting. We first consider the case where $g$ is analytic. Let $y \in M$ and introduce Riemannian normal coordinates $x=$ $\left(x^{0}, \ldots, x^{D-1}\right) \in \mathbb{R}^{D}$ about $y$. In this neighborhood of $y$, consider the 1-parameter family of metrics $g^{(s)}$ for all $|s| \leq 1$ defined by

$$
g^{(s)}=g_{\mu v}(s x) d x^{\mu} d x^{v} .
$$

Note that this family, in effect, interpolates between the given metric $g=g^{(1)}$ and the flat Minkowski metric $\eta=g^{(0)}$. We can expand $g^{(s)}$ in a power series in $s$ about $s=0$, which takes the form

$$
g_{\mu v}^{(s)}=\eta_{\mu v}+\sum_{n=2}^{\infty} s^{n} p_{\mu v \beta_{1} \ldots \beta_{n-2}}(y) x^{\beta_{1}} \ldots x^{\beta_{n-2}}
$$

where each $p$ is a curvature polynomial $p(y)=p\left[R_{\mu v \sigma \rho}(y), \ldots, \nabla_{\left(\alpha_{1}\right.} \cdots \nabla_{\alpha_{(n-2)}} R_{\mu v \sigma \rho}(y)\right]$. It can then be shown, using axiom (C1), that each OPE coefficient has an asymptotic expansion of the form

$$
C_{(j)}^{\left(i_{1}\right) \ldots\left(i_{n}\right)}\left(x_{1}, \ldots, x_{n}, y\right)=\sum_{k=0}^{\infty} q_{k}(y) \cdot\left(W_{k}\right)_{(j)}^{\left(i_{1}\right) \ldots\left(i_{n}\right)}\left(x_{1}, \ldots, x_{n}\right)
$$

where $q_{k}=\left(q_{k}\right)_{\mu_{1} \ldots \mu_{k}}$ is a curvature polynomial of the same general form as the $p$, and where $W_{k}=\left(W_{k}\right)^{\mu_{1} \ldots \mu_{k}}$ are distributions defined on a neighborhood of 0 in $\left(\mathbb{R}^{D}\right)^{n}$, valued in the tensor product of $\left(\mathbb{R}^{D}\right)^{\otimes k}$ with the spinor representation corresponding to the index structure of the quantum fields in the operator product considered. They transform covariantly under the connected component $\operatorname{Spin}_{\mathbb{R}}(D-1,1)_{0}$ of the spin group of $D$-dimensional Minkowski space.

Consider now the map $\rho$ defined in a suitable convex normal neighborhood, $O$, of $y$ by $\left(x^{0}, \ldots, x^{D-1}\right) \mapsto\left(-x^{0}, \ldots,-x^{D-1}\right)$. In Minkowski spacetime, this map would define an isometry which preserves spacetime orientation but reverses time orientation. In a general curved spacetime, this map does not define an isometry. Nevertheless, we may view $\rho$ as a map

$$
\rho:\left(O, g^{(s)}, e, T\right) \rightarrow\left(O, g^{(-s)}, e,-T\right) .
$$

Viewed in this manner, it is easily seen that $\rho$ preserves all background structure, i.e., it is a causality preserving isometry that preserves orientations. Consequently, by the covariance axiom (C1), the relation (64) is equivalent to a corresponding relation between the OPE-coefficients on the spacetimes $\left(M, g^{(s)}, e, T\right)$ and $\left(M, g^{(-s)}, e, T\right)$, i.e., spacetimes with different metrics but the same orientation and time orientation. If one now differentiates this relation $m$-times with respect to $s$ and puts $s=0$ afterwards, then one can prove that (64) is 
equivalent to the relation

$$
\begin{aligned}
& W_{k}\left(x_{1}, \ldots, x_{n}\right)= \\
& (-1)^{k} \pi^{*} W_{k}\left(-x_{n}, \ldots,-x_{1}\right) \cdot \begin{cases}\mathrm{i}^{-F(j)}(-1)^{-U(j)} \prod_{k=1}^{n} \mathrm{i}^{F\left(i_{k}\right)}(-1)^{U\left(i_{k}\right)} & m \text { even, } \\
\mathrm{i}^{-F(j)-U(j)+P(j)} \prod_{k=1}^{n} \mathrm{i}^{F\left(i_{k}\right)-U\left(i_{k}\right)+P\left(i_{k}\right)} & m \text { odd, }\end{cases}
\end{aligned}
$$

for all $k=0,1,2, \ldots$ Here, $\pi$ is the permutation (17), which acts by permuting the implicit spinor/indices associated with the spacetime points $x_{i}$.

We have thus reduced the proof of (64) to the proof of a statement about Minkowski distributions $W_{k}$ that transform covariantly under $\operatorname{Spin}_{\mathbb{R}}(D-1,1)_{0}$. To prove it, one next shows that $W_{k}$ can be analytically continued, and that the analytic continuation transforms covariantly under connected component of the identity in the complexified spin group $\operatorname{Spin}_{\mathbb{C}}(D-1,1)_{0}$. For this, one first proves, using the microlocal condition on the OPEcoefficients, that, near 0 , the analytic wave front set [22], $\mathrm{WF}_{A}$, of $W_{k}$ satisfies

$$
\mathrm{WF}_{A}\left(W_{k}\right) \subset K \text {. }
$$

Here, $K$ is a conic set defined in terms of the Minkowskian metric $\eta$ and orientation $e, T$, by

$$
\begin{aligned}
K= & \left\{\left(y_{1}, k_{1} ; \ldots ; y_{n}, k_{n}\right) \in T^{*}\left(\times{ }^{n} B_{r}\right) \backslash\{0\} \mid \exists p_{i j} \in \bar{V}_{+}, n \geq j>i \geq 1:\right. \\
& \left.k_{i}=\sum_{j: j>i} p_{i j}-\sum_{j: j<i} p_{j i} \text { for all } i\right\},
\end{aligned}
$$

where $\bar{V}^{+}$is the closure of the forward light cone $V^{+}$in Minkowski space (defined with respect to the time orientation $T$ ),

$$
V^{ \pm}=\left\{k \in \mathbb{R}^{D} \mid \eta_{\mu v} k^{\mu} k^{\nu}>0, \quad k^{\mu} \nabla_{\mu} T>0\right\} .
$$

The relation (72) is important because a theorem of [22] now guarantees that $W_{k}$ is the distributional boundary value

$$
W_{k}\left(x_{1}, \ldots, x_{n}\right)=\underset{\left(y_{1}, \ldots, y_{n}\right) \in K^{\mathrm{v}} \rightarrow 0}{\mathrm{~B} . \mathrm{V}} W_{k}\left(x_{1}+\mathrm{i} y_{1}, \ldots, x_{n}+\mathrm{i} y_{n}\right)
$$

of a holomorphic function $W_{k}\left(z_{1}, \ldots, z_{n}\right)$ that is defined in the "half-space"

$$
W_{k}: B_{r}(0)^{n}+\mathrm{i} K^{\mathrm{v}} \rightarrow \mathbb{C}, \quad \text { some } r>0
$$

where $B_{r}(0)$ is a ball of radius $r$ in $\mathbb{R}^{D}$, where $K^{\mathrm{v}}$ is the "dual cone" of all covectors $\left(y_{1}, \ldots, y_{n}\right) \in\left(\mathbb{R}^{D}\right)^{n}$ with the property that $\sum k_{i} \cdot y_{i}>0$ for all $\left(k_{1}, \ldots, k_{n}\right) \in K$. Using the "edge of the wedge-theorem" [27], one proves that the holomorphic function $W_{k}\left(z_{1}, \ldots, z_{n}\right)$ transforms covariantly under the spin group $\operatorname{Spin}_{\mathbb{R}}(D-1,1)_{0}$. As explained in more detail in [16], one can use this in turn to prove the desired relation (71):

For $D=2 m$ and $m$ even, we consider the chirality element $\Gamma$ in eq. (66) in flat space, which is an element of the connected component of the identity of the complexified spin 
group $\operatorname{Spin}_{\mathbb{C}}(D-1,1)_{0}$. It corresponds to the reflection element $\rho: \mathbb{R}^{D} \rightarrow \mathbb{R}^{D}, x \mapsto-x$ of the complexified Lorentz group $S O(D-1,1 ; \mathbb{C})$ under the standard covering homomorphism between these groups. This is an immediate consequence of the relation $\Gamma \gamma_{a} \Gamma^{-1}=-\gamma_{a}$. Using the method of analytic continuation in overlapping patches, it can be shown that $W_{k}$ may be continued to a single valued analytic function on an extension of the domain in eq. (76), and it can be shown that this continuation transforms covariantly under $\Gamma$. As explained above, $\Gamma$ acts as $+i d_{S_{+}}$on each tensor factor corresponding to a primed spinor index, and as $-i d_{S_{-}}$on each tensor factor associated with an unprimed spinor index. Therefore, if we apply the transformation law of $W_{k}$ under the element $\Gamma$, then we obtain relation (71) for complex spacetime arguments, except that the order of the complex spacetime arguments $z_{1}, \ldots, z_{n}$ is reversed, and except for the factors relating to the Bose-Fermi character of the fields involved. In order to be able to take the $\operatorname{limit} \operatorname{Im} z_{i} \rightarrow 0$ from within $K^{\mathrm{v}}$, we must pass to so-called "Jost points" $\left(z_{1}, \ldots, z_{n}\right)$ in the extended domain of holomorphicity. For such points, the (anti-)commutativity may be used, effectively allowing to permute the spacetime arguments in $W_{k}$ in such a way that one can take the limit to real points from within $K^{\mathrm{v}}$ as required in eq. (75) afterwards. When permuting the arguments, we pick up the factors related to the Bose/Fermi character of the fields.

For $D=2 m$ and $m$ odd, we consider instead the element i $\Gamma$ of the connected component of the identity of the complexified spin group $\operatorname{Spin}_{\mathbb{C}}(D-1,1)_{0}$. This element again covers the reflection $\rho(x)=-x$ on $D$-dimensional Minkowski space. It acts as $+\mathrm{i} i d_{S_{+}}$on each tensor factor corresponding to a primed spinor index, and as $-\mathrm{i} i d_{S_{-}}$on each tensor factor associated with an unprimed spinor index. Again it can be shown that $W_{k}$ may be continued analytically to a domain extending that in eq. (76), and that it transforms covariantly under $\mathrm{i} \Gamma$ on the extended domain. The additional factor of $\mathrm{i}$ gives rise to the different factors in eq. (71) compared to the case when $m$ is even. The rest of the argument is identical to that case.

This proves the PCT-relation (64) for analytic spacetimes, and even dimensions $D=$ $2 m$. The validity of the corresponding relation for smooth spacetimes then follows from the smoothness of the OPE-coefficients under smooth variations of the metric, since any smooth metric can be viewed as the limiting member of a smooth 1-parameter family of metrics $g^{(\lambda)}$ that are analytic for $\lambda>0$ and smooth for $\lambda=0$. The differences in the statement and proof of the PCT-relation (64) for odd spacetime dimension $D$ are described in remark (2) below, following the proof of the PCT-Theorem.

We now are ready to state and prove the spin-statistics theorem within our framework. The statement and proof of this theorem closely parallel the Minkowski spacetime version:

Theorem 2. (Spin-Statistics Theorem) If our axioms hold, then the spin statistics relation

$$
F(i)=U(i)+P(i) \quad \bmod 2,
$$

also holds, i.e. fields with integer spin (= on half the number of primed + unprimed spinor indices) have Bose statistics, while fields of half integer spin have Fermi statistics.

Proof: Let $i \in I$, and, as above, we restrict consideration to the even dimensional case $D=2 m$. Consider the PCT-relation (64) for the OPE-coefficient $C_{(\mathbf{1})}^{(i)\left(i^{\star}\right)}$. This condition can 
be written as

$$
C_{(\mathbf{1})}^{(i)\left(i^{\star}\right)}\left(x_{1}, x_{2}, y\right)_{\mathbf{M}} \sim C_{(\mathbf{1})}^{\left(i^{\star}\right)(i)}\left(x_{2}, x_{1} ; y\right)_{\overline{\mathbf{M}}} \cdot \begin{cases}\mathrm{i}^{F(i)+F\left(i^{\star}\right)}(-1)^{U\left(i^{\star}\right)+U(i)} & m \text { even }, \\ \mathrm{i}^{F(i)+U(i)-P(i)+F\left(i^{\star}\right)+U\left(i^{\star}\right)-P\left(i^{\star}\right)} & m \text { odd },\end{cases}
$$

where we have used the hermitian conjutation axiom, and where we have used that $F(\mathbf{1})=0$ since the identity is always a Bose field, by the identity axiom. When $m$ is even, then $U\left(i^{\star}\right)=P(i)$ because conjugation of a spinor exchanges the number of primed and unprimed indices. Furthermore, $F(i)=F\left(i^{\star}\right)$ by Theorem 1, so we obtain

$$
C_{(\mathbf{1})}^{(i)\left(i^{\star}\right)}\left(x_{1}, x_{2}, y\right)_{\mathbf{M}} \sim(-1)^{F(i)+U(i)+P(i)} C_{(\mathbf{1})}^{\left(i^{\star}\right)(i)}\left(x_{2}, x_{1} ; y\right) \overline{\mathbf{M}} .
$$

When $m$ is odd, $U\left(i^{\star}\right)=U(i)$ and $P\left(i^{\star}\right)=P(i)$, because conjutation of a spinor does not change the number of primed and unprimed spinor indices in that case. Using this, we again obtain the expression eq. (79) when $m$ is odd.

We now smear this expression with the test section $f_{\lambda}\left(x_{1}\right) \bar{f}_{\lambda}\left(x_{2}\right)$, where

$$
f_{\lambda}(x)=\lambda^{-D} f\left[y+\lambda^{-1}(x-y)\right] .
$$

We are taking here an $f$ of compact support in a sufficiently small neighborhood of $y$ covered by some coordinate system, and $y+\lambda^{-1}(x-y)$ is computed in this arbitrary coordinate system. Denote by $\operatorname{dim}(i)$ the dimension of the field labelled by $i$. It follows from eq. (79) that

$$
\begin{aligned}
\lambda^{2 \operatorname{dim}(i)-\delta} \int C_{(\mathbf{1})}^{(i)\left(i^{\star}\right)}\left(x_{1}, x_{2} ; y\right) & \mathbf{M} f_{\lambda}\left(x_{1}\right) \bar{f}_{\lambda}\left(x_{2}\right) d \mu_{1} d \mu_{2} \\
-p \lambda^{2 \operatorname{dim}(i)-\delta} \int C_{(\mathbf{1})}^{\left(i^{\star}\right)(i)}\left(x_{2}, x_{1} ; y\right) & \overline{\mathbf{M}}_{\lambda}\left(x_{1}\right) \bar{f}_{\lambda}\left(x_{2}\right) d \mu_{1} d \mu_{2} \rightarrow 0
\end{aligned}
$$

as $\lambda \rightarrow 0$, where $p=(-1)^{F(i)+U(i)+P(i)}$. But the first term goes to $+\infty$ by lemma 2 , while the second term goes to $-p \cdot \infty$ for suitable $f$. Thus, these terms can only cancel for small $\lambda$ if we have $p=+1$, meaning that $F(i)+P(i)+U(i)=0$ modulo 2 . Hence the spin-statistics relation must hold.

Next, we state and prove the PCT theorem, the formulation of which is quite different from the Minkowski spacetime version (see the discussion below). Again, we restrict consideration here to $D=2 m$, and describe the differences occurring in odd dimensions in remark (2) below:

Theorem 3. (PCT-Theorem) Given a background structure $\mathbf{M}=(M, g, e, T)$, we write $\overline{\mathbf{M}}=$ $(M, g, e,-T)$. In spacetime dimension $D=2 m$, define the anti-linear map $\theta_{\mathbf{M}}^{\mathrm{PCT}}: \mathcal{A}(\mathbf{M}) \rightarrow$ $\mathcal{A}(\overline{\mathbf{M}})$ by

$$
\theta_{\mathbf{M}}^{\mathrm{PCT}}: \phi_{\mathbf{M}}^{(i)}(f) \mapsto \phi_{\overline{\mathbf{M}}}^{(i)}(f)^{*} \cdot \begin{cases}\mathrm{i}^{F(i)}(-1)^{U(i)} & \text { when } m \text { is even, } \\ \mathrm{i}^{F(i)+U(i)-P(i)} & \text { when } m \text { is odd. }\end{cases}
$$


Then $\theta_{\mathbf{M}}^{\mathrm{PCT}}$ is an anti-linear *-isomorphism such that the diagram

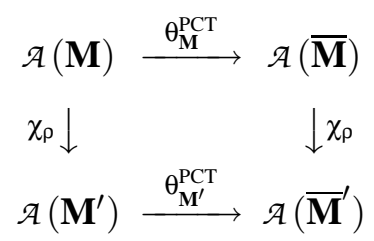

as well as the diagram

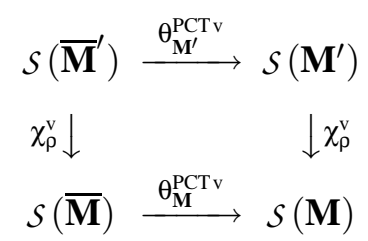

commute for every isometric, causality and orientation preserving embedding $\rho: \mathbf{M} \rightarrow \mathbf{M}^{\prime}$. Here $\chi_{\rho}^{\mathrm{v}}$ denotes the dual of the linear map $\chi_{\rho}$, and $\theta^{\mathrm{PCT} v}$ denotes the dual of $\theta^{\mathrm{PCT}}$.

Proof: The proof of this theorem is, in essence, an application of lemma1 In the notation of lemma 11, we choose $\mathbf{M}=(M, g, e, T)$, we choose $\mathbf{M}^{\prime}=\overline{\mathbf{M}}$, we take $\psi=i d$ and we choose $L: i \mapsto i^{\star}$. For any index $i$, we define $\psi_{(i)}$ to be the composition of the natural anti-linear bundle map from $V_{\mathbf{M}}(i)$ to $V_{\overline{\mathbf{M}}}\left(i^{\star}\right)$ that is implicit in the formula (82) with the multiplication map by $\mathrm{i}^{F(i)}(-1)^{U(i)}$ when $m$ is even and by $\mathrm{i}^{F(i)+U(i)-P(i)}$ when $m$ is odd. From this definition we then have

$$
\operatorname{conj}_{\overline{\mathbf{M}}} \circ \psi_{(i)}= \begin{cases}(-1)^{F(i) / 2+F\left(i^{\star}\right) / 2+U(i)-U\left(i^{\star}\right)} \cdot \Psi_{\left(i^{\star}\right)} \circ \operatorname{conj}_{\mathbf{M}} & m \text { even, } \\ \mathrm{i}^{F(i)+F\left(i^{\star}\right)-U(i)-U\left(i^{\star}\right)+P(i)+P\left(i^{\star}\right)} \cdot \Psi_{\left(i^{\star}\right)} \circ \operatorname{conj}_{\mathbf{M}} & m \text { odd, }\end{cases}
$$

where $\operatorname{conj} j_{\mathbf{M}}$ is the anti-linear map that sends a spinor to the hermitian conjugate spinor on $M$. Now for even $m$ the number of unprimed spinor indices associated with $V(i)$ is precisely equal to the number of primed indices $P\left(i^{\star}\right)$ associated with $V\left(i^{\star}\right)$, because $V\left(i^{\star}\right)$ is assumed to be equal to $\bar{V}(i)$. Thus, $P(i)=U\left(i^{\star}\right)$. Furthermore, we have $F(i)=F\left(i^{\star}\right)$ by Theorem 1 , and $F(i)=U(i)+P(i) \bmod 2$ by the spin-statistics theorem. Consequently, the compatibility condition for the *-operation holds when $m$ is even, i.e.,

$$
\operatorname{conj}_{\overline{\mathbf{M}}} \circ \psi_{(i)}=\psi_{\left(i^{\star}\right)} \circ \operatorname{conj}_{\mathbf{M}} .
$$

When $m$ is odd, then $U(i)=U\left(i^{\star}\right)$ and $P(i)=P\left(i^{\star}\right)$, and the compatibility condition again holds because of $F(i)=F\left(i^{\star}\right)$ and the spin-statistics theorem. Thus, we have shown that the first input in lemma 1 holds. The second input is the compatibility of the OPE coefficients on $\mathbf{M}$ and $\mathbf{M}^{\prime}$, eq. (44). That condition is essentially equivalent to the relation (64), except that the latter relation also involves an additional complex conjugation of the OPEcoefficient. However it is immediately seen that this will result only in the following difference in the conclusion of lemma 1; Instead of the linear *-homomorphism as provided by this lemma, we now find that the PCT-map $\theta^{\text {PCT }}$ defined by eq. (82) yields an anti-linear *-homomophism. 
Remarks: (1) The above formulation of the PCT-theorem was suggested in [16]. As noted in [9] the theorem can be stated in the language of functors by saying that the functors $\mathbf{M} \rightarrow \mathcal{A}(\mathbf{M})$ and $\mathbf{M} \rightarrow \overline{\mathcal{A}}(\mathbf{M})=\mathcal{A}(\overline{\mathbf{M}})$ are equivalent.

(2) In odd spacetime dimensions $D=2 m+1$, the chirality operator $\Gamma$ of eq. (66) is proportional to the identity in $S$. Thus, in this case there is no decomposition $S=S_{+} \oplus S_{-}$as in the even dimensional case, and there is consequently no difference between "primed" and "unprimed" spinors. If we denote the number of spinor indices of a quantum field by $N(i)$ (i.e., the bundle associated with the label $i$ the field is $\left.V(i)=S^{\otimes N(i)}\right)$, then the factors in formula (64) are now $\mathrm{i}^{-F(j)-N(j)} \prod \mathrm{i}^{F\left(i_{k}\right)+N\left(i_{k}\right)}$. In the proof of this formula, one must now consider the map $\rho:\left(x^{0}, x^{1}, \ldots, x^{D-1}\right) \mapsto\left(-x^{0},-x^{1}, \ldots,+x^{D-1}\right)$. This corresponds again to a change of time orientation in Minkowski spacetime which leaves the spacetime orientation invariant. The rest of the proof is similar.

We now explain the relation of the above formulation of the PCT theorem to to the usual PCT theorem in Minkowski spacetime (see e.g. [27]). Changing $T \rightarrow-T$ while keeping $e$ unchanged is equivalent to changing parity (i.e., the spatial orientation $s$ of a Cauchy surface $\Sigma$ induced by $e$ and $T$ via $d T \wedge s=e$ ) and time (i.e., the time function). Furthermore, the field appearing on the right side of eq. (82) is usually referred to as the "charge conjugate field" to $\phi_{\mathbf{M}}^{(i)}(f)$. Thus our formulation of the PCT theorem asserts that the theory is indeed invariant under simultaneous PCT-reversal in the sense that the theory on $\mathbf{M}$ is "the same" as the theory on $\overline{\mathbf{M}}$ with the fields replaced by their charge conjugates. However, note that our PCT theorem relates theories on the two different background structures, $\mathbf{M}$ and $\overline{\mathbf{M}}$. By contrast, the usual PCT theorem in Minkowski spacetime provides a symmetry of the theory defined on a single background structure, namely Minkowski spacetime with a fixed choice of orientation and time orientation. Indeed, the usual formulation of the PCT theorem in Minkowski spacetime asserts the existence of an anti-unitary operator $\Theta: \mathcal{H} \rightarrow \mathcal{H}$ on the Hilbert space, $\mathcal{H}$, of physical states such that, if $\rho$ denotes the isometry on the, say, evendimensional Minkowski spacetime defined by

$$
\rho:\left(x^{0}, x^{1}, \ldots, x^{D-1}\right) \mapsto\left(-x^{0},-x^{1}, \ldots,-x^{D-1}\right),
$$

and if $\phi^{C}$ is the charge conjugate field associated with $\phi$ defined by eq. (82), then $\operatorname{Ad}_{\Theta} \phi(\rho(x)) \equiv$ $\Theta \phi(\rho(x)) \Theta^{\dagger}=\phi^{C}(x)$.

The relationship between these formulations can be seen as follows. Start with our formulation of the PCT theorem. The isometry $\rho$ maps Minkowski spacetime $\mathbf{M}=\left(\mathbb{R}^{D}, \eta, e, T\right)$ with a given choice of orientation $e$ and time orientation $T$, to $\overline{\mathbf{M}}=\left(\mathbb{R}^{D}, \eta, e,-T\right)$. Thus, by "application 1" of lemma1, we know that there is a *homomorphism $\chi_{\rho}: \mathcal{A}(\mathbf{M}) \rightarrow \mathcal{A}(\overline{\mathbf{M}})$ mapping the quantum fields $\phi_{\mathbf{M}}(f)$ on $\mathbf{M}$ to the "same" quantum fields $\phi_{\overline{\mathbf{M}}}\left(\rho_{*}(f)\right)$ on $\overline{\mathbf{M}}$. Thus, if we define

$$
\operatorname{Ad}_{\Theta}:=\chi_{\rho}^{-1} \circ \theta_{\mathbf{M}}^{\mathrm{PCT}} .
$$

we obtain a result that is essentially equivalent to the usual Minkowski version (as suitably reformulated in an algebraic setting). Conversely, if we start with the usual formulation of the PCT theorem and if we define quantum field theory on $\mathcal{A}(\overline{\mathbf{M}})$ in terms of quantum field 
theory on $\mathbf{M}$ by means of the map $\chi_{\rho}$, then we obtain a version essentially equivalent to our formulation by setting

$$
\theta_{\mathbf{M}}^{\mathrm{PCT}}=\chi_{\rho} \circ \operatorname{Ad}_{\Theta} .
$$

Although the above formulations are essentially equivalent in Minkowski spacetime, in a general spacetime, there does not exist any discrete isometry analogous to $\rho$. Thus, in general we only have a PCT theorem describing the relation between the theory defined on different backgrounds $\mathbf{M}$ and $\overline{\mathbf{M}}$. Of course in the case of a spacetime that admits an isometry $\rho$ mapping $(e, T)$ to $(e,-T)$ (as occurs, e.g. in Schwarzschild and deSitter spacetimes), then a "same background structure" version of the PCT theorem can be given via eq. (88).

The example of a Robertson-Walker spacetime

$$
g=-d t^{2}+a(t)^{2} d \mathbf{x} \cdot d \mathbf{x}
$$

with $a(t)$ a strictly increasing function of $t$, may be useful for clarifying the physical meaning of our formulation of the PCT theorem in a general curved spacetime. If we choose the time orientation $T=t$, then the above metric describes an expanding universe, while if we take $T=-t$, it describes a corresponding contracting universe (with opposite choice of spatial orientation since we keep $e$ fixed). In essence, our formulation of the PCT theorem relates phenomena/processes occuring in the expanding universe eq. 90) to corresponding processes (involving the charge conjugate fields and also a reversal of parity) in the corresponding contracting universe. Since the metric eq. (90) has no time reflection isometry $\rho$, there are no relations implied by the PCT theorem between phenomena/processes occurring in the expanding universe, eq. (90) with $T=t$. As a concrete illustration of this, suppose that it were possible to give a definition of "particle masses" in curved spacetime-although it is far from obvious that any such useful notion exists. The PCT theorem would then imply that the mass of a particle in an expanding universe must be equal to the mass of the corresponding antiparticle in a contracting universe. However, it would make no statement about the masses of particles and antiparticles in the same universe 13 .

Finally, it is worth emphasizing the nature of the action of our PCT map $\theta_{\mathbf{M}^{\prime}}^{\mathrm{PCT}}$ on states. In Minkowski spacetime, the states of interest are normally assumed to lie in a single Hilbert space $\mathcal{H}$, and one often considers scattering states. In the usual formulation of the PCT theorem in Minkowski spacetime, if $\omega$ describes an incoming scattering state $\mid p_{1}, \ldots, p_{n} ;$ in $\rangle \in \mathcal{H}$, then the corresponding state $\bar{\omega}$ under the PCT map may be identified with an outgoing scattering state $\mid-p_{1}, \ldots,-p_{n}$; out $\rangle \in \mathcal{H}$ in the same Hilbert space. However, in our formulation of the PCT theorem, if $\omega$ is an in-state in $\mathbf{M}$, then $\bar{\omega}$ is an in-state in $\overline{\mathbf{M}}$. If the spacetime admits a time reflection isometry $\rho$, then $\bar{\omega}$ may be identified with

\footnotetext{
${ }^{13}$ It is worth noting that the "third Sakharov necessary condition" for baryogenesis in the early universe (namely, "interactions out of thermal equilibrium") is based upon the (now seen to be unjustified) assumption that particle and antiparticle masses are equal in an expanding universe. However, to the extent that particle and antiparticle masses might differ in an expanding universe (even assuming that a useful notion of "particle mass" can be defined) as a result of the lack of a time reflection symmetry, it would probably not even be possible to define a notion of "thermal equilibrium" as a result of the lack of a time translation symmetry.
} 
a corresponding out-state on M. However, for the Robertson-Walker spacetime eq. (90), which does not admit a time reflection isometry, the PCT theorem relates in-states in an expanding universe to in-states in the corresponding contracting universe.

\section{Conclusions and outlook}

In this paper we have proposed a new axiomatic framework for quantum field theory on curved spacetime. We demonstrated that our new framework captures much of the same content as the Wightman axioms by proving curved spacetime analogs of the spin-statistics theorem and PCT theorem. In this section, we discuss some of the implications and potential ramifications of the viewpoint suggested by this new framework.

First, we address the issue of why we even seek an axiomatic framework for quantum field theory in curved spacetime at all. Since gravity is being treated classically, quantum field theory in curved spacetime cannot be a fundamental theory of nature, i.e., it must have a limited domain of validity. In particular, we have focused considerable attention in this paper on the OPE of quantum fields in curved spacetime, but the OPE is a statement about the arbitrarily-short-distance singularity structure of products of fields. One would not expect that quantum field theory in curved spacetime would give an accurate description of nature at separations smaller than, say, the Planck scale. Consequently, why should one seek a set of mathematically consistent rules governing quantum field theory that are rigorously applicable only in a regime where the theory is not expected to be valid?

Our response to this question is that an exactly similar situation arises for classical field theory. Classical field theory also is not a fundamental theory of nature, and its description of nature makes essential use of differentiability/smoothness properties of the classical fields at short distance scales; one could not even write down the partial differential equations governing the evolution of classical fields without such short-distance-scale assumptions. However, if quantum field theory is any guide, the description of physical fields as smooth tensor fields is drastically wrong at short distance scales. Nevertheless, classical field theory has been found to give a very accurate description of nature within its domain of validity, and we have obtained a great deal of insight into nature by obtaining a mathematically precise formulation of classical field theory. It is our belief that there exists a mathematically consistent framework for quantum field theory in curved spacetime, and that by obtaining and studying this framework, we will not only get an accurate description of nature within the domain of validity of this theory, but we will also get important insights and clues concerning the nature of quantum gravity.

We began our quest for the mathematical framework of quantum field theory in curved spacetime by seeking to generalize the Wightman axioms to curved spacetime in as conservative a manner as possible. As described much more fully in the Introduction, there are three key ingredients of the Wightman axioms that do not generalize straightforwardly to curved spacetime: (1) Poincare invariance; (2) the spectrum condition; (3) existence of a Poincare invariant state. We have seen in this paper that quantum field theory can be generalized to curved spacetime by replacing these ingredients by the following: (1') quantum fields are locally and covariantly defined; (2') the microlocal spectrum condition; (3') 
existence of an OPE. Although the formulation of conditions (1') and (2') differs significantly from the formulation of conditions (1) and (2), the basic content of these conditions is essentially the same. Indeed, there would be no essential difference in the formulation of axiomatic quantum field theory in Minkowski spacetime if one replaced (1) and (2) with (1') and (2'). By contrast, as we shall elucidate further below, the replacement of (3) by (3') leads to a radically different viewpoint on quantum field theory.

The most important aspect of this difference is that the existence of a "preferred state" no longer plays any role in the formulation of the theory. States are inherently non-local in character, and the replacement of (3) by (3') - along with the replacements of (1) with (1') and (2) with (2') - yields a formulation of quantum field theory that is entirely local in nature. In this way, the formulation of quantum field theory becomes much more analogous to the formulation of classical field theory. Indeed, one can view a classical field theory as being specified by providing the list of fields $\phi^{(i)}$ occuring in the theory and the list of local, partial differential relations satisfied by these fields. Solutions to the classical field theory are then sections of the appropriate vector bundles that satisfy the partial differential relations as well as regularity conditions (e.g., smoothness). Similarly, in our framework, a quantum field theory is specified by providing the list of fields $\phi^{(i)}$ occuring in the theory and the list of local, OPE relations satisfied by these fields. Thus, the OPE relations play a role completely analogous to the role of field equations in classical field theory. Stateswhich are the analogs of solutions in classical field theory-are positive linear maps on the algebra $\mathcal{A}$ defined in section 3 that satisfy the OPE relations as well as regularity conditions (in this case, the microlocal spectrum condition). We note that in classical field theory, the field equations always manifest all of the symmetries of the theory, even in cases where there are no solutions that manifest these symmetries. Similarly, in our formulation of quantum field theory, the OPE relations that define the theory should always respect the symmetries of the theory [30], even if no states happen to respect these symmetries.

Our viewpoint on quantum field theory is more restrictive than standard viewpoints in that we require the existence of an OPE. On the other hand, it is less restrictive in that we do not require the existence of a ground state. This latter point is best illustrated by considering a free Klein-Gordon field $\varphi$ in Minkowski spacetime

$$
\left(\square-m^{2}\right) \varphi=0,
$$

where the mass term, $m^{2}$, is allowed to be positive, zero, or negative. In the standard viewpoint, a quantum field theory of the free Klein-Gordon field does not exist in any dimension when $m^{2}<0$ and does not exist in $D=2$ when $m^{2}=0$ on account of the nonexistence of a Poincare invariant state. However, there is no difficulty is specifying OPE relations that satisfy our axioms for all values of $m^{2}$ and all $D \geq 2$. In particular, for $D=4$ we can choose the OPE-coefficient $C$ of the identity in the OPE of $\varphi\left(x_{1}\right) \varphi\left(x_{2}\right)$ to be given by

$$
\begin{aligned}
& C\left(x_{1}, x_{2} ; y\right)= \\
& \frac{1}{4 \pi^{2}}\left(\frac{1}{\Delta x^{2}+i 0 t}+m^{2} j\left[m^{2} \Delta x^{2}\right] \log \left[\mu^{2}\left(\Delta x^{2}+i 0 t\right)\right]+m^{2} h\left[m^{2} \Delta x^{2}\right]\right),
\end{aligned}
$$


where $\Delta x^{2}=\left(x_{1}-x_{2}\right)^{2}$ and $t=x_{1}^{0}-x_{2}^{0}$. Here $\mu$ is an arbitrarily chosen mass scale and $j(z) \equiv \frac{1}{2 i \sqrt{z}} J_{1}(i \sqrt{z})$ is an analytic function of $z$, where $J_{1}$ denotes the Bessel function of order 1. Furthermore, $h(z)$ is the analytic function defined by

$$
h(z)=-\pi \sum_{k=0}^{\infty}[\psi(k+1)+\psi(k+2)] \frac{(z / 4)^{k}}{k !(k+1) !} .
$$

with $\psi$ the psi-function. This formula for the OPE coefficient—as well as the corresponding formulas for all of the other OPE coefficients-is as well defined for negative $m^{2}$ as for positive $m^{2}$. Existence of states satisfying all of the OPE relations for negative $m^{2}$ can be proven by the deformation argument of [12], using the fact that such states exist for positive $m^{2}$.

Although, in our framework, the Klein-Gordon field with negative $m^{2}$ now joins the ranks of legitimate quantum field theories, this theory is not physically viable because, in all states, field quantities will grow exponentially in time 14 . The potential importance of the above example is that it explicitly demonstrates that the local OPE coefficients can have a much more regular behavior under variations of the parameters of the theory as compared with state-dependent quantities, such as vacuum expectation values. The OPE coefficients in the above example are analytic in $\mathrm{m}^{2}$. On the other hand, the 2-point function of the global vacuum state is, of course, defined only for $m^{2} \geq 0$ and is given by

$$
\begin{aligned}
& \left\langle 0\left|\varphi\left(x_{1}\right) \varphi\left(x_{2}\right)\right| 0\right\rangle= \\
& \frac{1}{4 \pi^{2}}\left(\frac{1}{\Delta x^{2}+i 0 t}+m^{2} j\left[m^{2} \Delta x^{2}\right] \log \left[m^{2}\left(\Delta x^{2}+i 0 t\right)\right]+m^{2} h\left[m^{2} \Delta x^{2}\right]\right) .
\end{aligned}
$$

This behaves non-analytically in $m^{2}$ at $m^{2}=0$ on account of the $\log m^{2}$ term. In other words, in free Klein-Gordon theory, vacuum expectation values cannot be constructed perturbatively by expanding about $m^{2}=0$ - as should be expected, since no vacuum state exists for $m^{2}<0$ - but there is no difficulty in perturbatively constructing the OPE coefficients by expanding about $m^{2}=0$.

The above considerations raise the possibility that the well known failure of convergence of perturbation series in interacting quantum field theory may be due to the nonanalytic dependence of states on the parameters of the theory, rather than any non-analytic dependence of the fields themselves, i.e., that the OPE coefficients may vary analytically with the parameters of the theory. In other words, we are suggesting the possibility that the perturbation series for OPE coefficients may converge, and, thus, that, within our framework, it may be possible to perturbatively construc 15 interacting quantum field theories. In

\footnotetext{
${ }^{14}$ In this respect, the quantum field of the Klein-Gordon field with negative $m^{2}$ behaves very similarly to the corresponding classical theory. The classical Klein-Gordon field with negative $m^{2}$ has a well posed initial value formulation and causal propagation (despite frequently expressed claims to the contrary). However, the classical Klein-Gordon field with negative $m^{2}$ is not physically viable since it is unstable, i.e., it admits solutions that grow exponentially with time.

${ }^{15}$ However, we are not suggesting that it should be possible to perturbatively construct states of the theory. Even if one had the complete list of OPE coefficients, it would not be obvious how to construct states.
} 
order to do so, it will be necessary to define the basis fields $\phi^{(i)}$ appropriately (see below and [18]) and also to parametrize the theory appropriately (since a theory with an analytic dependence on a parameter could always be made to appear non-analytic by a non-analytic reparametrization). Aside from the free Klein-Gordon example above, the only evidence we have in favor of convergence of perturbative expansions for OPE coefficients is the example of super-renormalizable theories, such as $\lambda \varphi^{4}$-theory in two spacetime dimensions [17]. Here, only finitely many terms in a perturbative expansion can contribute to any OPE coefficient up to any given scaling degree, so convergence (up to any given scaling degree) is trivial. By contrast, for $\lambda \varphi^{4}$-theory in two spacetime dimensions, the rigorously constructed, non-perturbative ground state $n$-point functions can be proven to be non-analytic at $\lambda=0[25]$.

In cases-such as free Klein-Gordon theory above-where the OPE coefficients can be chosen to be analytic in the parameters of the theory, it seems natural to require that the theory be defined so that this analytic dependence holds. This requirement has some potentially major ramifications. Since vacuum expectation values of a products of fields (i.e., a correlation function) would be expected to have a non-analytic dependence on the parameters of the theory, it follows that if the OPE coefficients have an analytic dependence on these parameters, then, even in Minkowski spacetime, some of the fields appearing on the right side of the OPE of a product of fields must acquire a nonvanishing vacuum expectation value, at least for some values of the parameters. This point is well illustrated by the above Klein-Gordon example. It is natural to identify the next term in the OPE of $\varphi\left(x_{1}\right) \varphi\left(x_{2}\right)$ [i.e., the term beyond the identity term, whose coefficient is given by eq. (92)] as being $\varphi^{2}$ (with unit coefficient), i.e.,

$$
\varphi\left(x_{1}\right) \varphi\left(x_{2}\right) \sim C\left(x_{1}, x_{2} ; y\right) \mathbf{1}+\varphi^{2}(y)+\ldots .
$$

This corresponds to the usual "point-splitting" definition of $\varphi^{2}$, except that $C\left(x_{1}, x_{2} ; y\right)$ now replaces $\left\langle 0\left|\varphi\left(x_{1}\right) \varphi\left(x_{2}\right)\right| 0\right\rangle$. If we take the vacuum expectation value of this formula (for $m^{2} \geq 0$, when a vacuum state exists) and compare it with eq. (94), we obtain

$$
\left\langle 0\left|\varphi^{2}(y)\right| 0\right\rangle=-\frac{m^{2}}{16 \pi^{2}} \log \left(m^{2} / \mu^{2}\right) .
$$

Thus, we cannot set $\left\langle 0\left|\varphi^{2}\right| 0\right\rangle=0$ for all values of $m^{2}$. A similar calculation for the stressenergy tensor of $\varphi$ yields

$$
\left\langle 0\left|T_{a b}(y)\right| 0\right\rangle=\frac{m^{4}}{64 \pi^{2}} \log \left(m^{2} / \mu^{2}\right) \eta_{a b}
$$

As in other approaches, the freedom to choose the arbitrary mass scale $\mu$ in eq. (92) gives rise to a freedom to choose the value of the "cosmological constant term" in eq. (97).

\footnotetext{
${ }^{16}$ The point-split expression using $\left\langle 0\left|\varphi\left(x_{1}\right) \varphi\left(x_{2}\right)\right| 0\right\rangle$ yields the "normal ordered" quantity : $\varphi^{2}:$. From the point of view of quantum field theory in curved spacetime it is much more natural define $\varphi^{2}$ via eq. 95) than by normal ordering, since there is no generalization of normal ordering to curved spacetime that is compatible with a local and covariant definition of $\varphi^{2}$ [19] Indeed, it follows from the results of [19] that eq. (95] is the unique way to define $\varphi^{2}$ compatible with desired properties, with the only ambiguities in the definition of $\varphi^{2}$ arising from different allowed choices of $C\left(x_{1}, x_{2} ; y\right)$.
} 
However, unlike other approaches, there is no freedom to adjust the value of the cosmological constant when $m^{2}=0$ (i.e., we unambiguously obtain $\left\langle 0\left|T_{a b}\right| 0\right\rangle=0$ in Minkowski spacetime in that case), and the $m^{2}$-dependence of the cosmological constant is fixed (since $\mu$ is not allowed to depend upon $m^{2}$ ).

A much more interesting possibility arises for interacting field theories, such as nonabelian gauge theories. In such theories, it is expected that there are "non-perturbative effects" that vary with the coupling parameter $g$ as $\exp \left(-1 / g^{2}\right)$. Such non-perturbative effects can potentially be very small compared with the natural scales appearing in the theory. If such non-perturbative terms occur the vacuum expectation values of products of fields and if - as we have speculated above - the OPE coefficients have an analytic dependence on the coupling parameter, then composite fields-such as the stress-energy tensor-must acquire nonvanishing vacuum expectation values that vary as $\exp \left(-1 / g^{2}\right)$. This possibility appears worthy of further investigation.

Acknowledgments: This research was supported in part by NSF Grant PHY04-56619 to the University of Chicago.

\section{A Definition of the scaling degree and wave front set of a distribution}

In this appendix, we recall the notion of scaling degree and of the wave front set of a distribution, which play an important role in the body of the paper. Quite generally, let $u$ be an distribution on $\mathbb{R}^{n}$. We say that $u$ has scaling degree $d$ at $x=0$, if $d$ is the smallest real number such that $\lambda^{\delta} u\left(f_{\lambda}\right) \rightarrow 0$ as $\lambda \rightarrow 0+$, for all $\delta>d$. Here, $f_{\lambda}(x)=\lambda^{-n} f\left(\lambda^{-1} x\right)$ denotes the function of compact support that is obtained by rescaling a smooth test function $f$ around $x=0$, making it more and more sharply peaked at that point. The scaling degree at an arbitrary point is obtained by simply translating the distribution $u$ or the test function $f$ by the desired amount. We write $\operatorname{sd}_{x}(u)=d$ for the scaling degree at a point $x$.

We next recall the definition of the wave front set of a distribution $u$ on $\mathbb{R}^{n}$. Let $\chi$ be any smooth function of compact support. Then $\chi u$ is evidently a distribution of compact support, and its Fourier transform $\widehat{\chi u}(k)$ defines an entire function of $k \in \mathbb{R}^{n}$. For any distribution $v$ of compact support, we define its corresponding "singular set", $\Sigma(v)$ as the collection of all $k \in \mathbb{R}^{n}$ such that

$$
|\widehat{v}(\lambda k)| \geq C \lambda^{N},
$$

for some $C>0$, and some $N$, and all $\lambda>0$. We define the wave front set $\mathrm{WF}_{x}(u)$ at a point $x \in \mathbb{R}^{n}$ as the intersection

$$
\mathrm{WF}_{x}(u)=\bigcap_{\chi: x \in \operatorname{supp} \chi} \Sigma(\chi u)
$$

and we define $\operatorname{WF}(u)$ as the union

$$
\mathrm{WF}(u)=\bigsqcup_{x \in \mathbb{R}^{n}} \mathrm{WF}_{x}(u) .
$$


Each set $\mathrm{WF}_{x}(u)$ is a conic set, in the sense that if $k \in \mathrm{WF}_{x}(u)$, then so is $t k$ for any $t>0$, and $k=0$ is never in $\mathrm{WF}_{x}(u)$. It immediately follows from the definition that $\mathrm{WF}_{x}(u)=\emptyset$ if and only if $u$ can be represented by a smooth function in an open neighborhood of $x$. In this sense, the wave-front set tells one at which points a distribution is singular. It also contains information about the most singular directions in local momentum space, which are represented by $k \in \mathrm{WF}_{x}(u)$.

It turns out that both the scaling degree of a distribution at a point $x$, as well as the wave front set at $x$ are invariantly defined. By this one means the following. Let $\rho: V \rightarrow$ $U$ be a smooth diffeomorphism between open sets $U, V \subset \mathbb{R}^{n}$. Let $u$ be a distribution supported in $U$, and let $\rho^{*} u$ be the pulled back distribution in $V$, where the pull-back is defined by analogy with the pull back of a smooth function. Then it is easy to show that $\operatorname{sd}_{x}\left(\rho^{*} u\right)=\operatorname{sd}_{\rho(x)}(u)$. Furthermore, if $x^{\prime}=\rho(x)$, and if we define $\rho^{*}\left(x^{\prime}, k^{\prime}\right)=(x, k)$, where $k=[d \rho(x)]^{\mathrm{v}} k^{\prime}$, then one can show

$$
\mathrm{WF}_{x}\left(\rho^{*} u\right)=\rho^{*} \mathrm{WF}_{\rho(x)}(u) .
$$

These relations imply that the scaling degree and the wave front set can be invariantly defined on an arbitrary manifold $X$, and that the wave-front set should be viewed as a subset of $T^{*} X$.

In the body of the paper, we frequently consider the case $X=M^{n+1}$, and the scaling degree at the point $(y, y, \ldots, y)$, i.e., points on the total diagonal. To save writing, this is simply denoted sd $u$.

\section{B Equivalent formulation of condition (C6)}

In this appendix, we relate the scaling degree (C5) and asymptotic positive (C6) assumptions to other properties of the quantum field theory. Our first is just a repetition of a result obtained in [8]:

Theorem 4. For any $x \in M$, and any (scalar) field $T$ not equal to a multiple of the identity operator, we define the convex set $S_{x} \subset \mathbb{R}$ by

$$
S_{x}=\left\{\langle T(x)\rangle_{\Phi} \mid \Phi \text { a normalized state }\right\}
$$

Then, $S_{x}=\mathbb{R}$ for at least one spacetime $\mathbf{M}$.

Remark: The statement means that pointlike hermitian fields $T(x)$ are unbounded from above and below, even though their classical counterpart (if the theory has a classical limit) might be manifestly non-negative, such as the Wick square $T=\varphi^{2}$, or the energy density $T=T_{a b} u^{a} u^{b}$ of a free Klein-Gordon field $\varphi$.

Proof: Choose any state $\Phi$. We may assume that $\langle T(f)\rangle_{\Phi}=0$, because if not, we just need to consider instead $T(x)$ by $T(x)-\langle T(x)\rangle_{\Phi} \mathbf{1}$. Define

$$
A=\cos \alpha \mathbf{1}+\sin \alpha \frac{T(f)}{\langle T(f) T(f)\rangle_{\Phi}^{1 / 2}},
$$


and define a new normalized state by $\langle.\rangle_{\alpha}=\left\langle A . A^{*}\right\rangle_{\Phi}$. Then

$$
\langle T(f)\rangle_{\alpha}=a \sin 2 \alpha+b(1-\cos 2 \alpha)
$$

where

$$
a=\langle T(f) T(f)\rangle_{\Phi}^{1 / 2}, \quad b=\frac{1}{2} \frac{\langle T(f) T(f) T(f)\rangle_{\Phi}}{\langle T(f) T(f)\rangle_{\Phi}} .
$$

Minimizing over $\alpha$ gives

$$
\inf _{\Psi}\langle T(f)\rangle_{\Psi} \leq b-\sqrt{a^{2}+b^{2}}
$$

Replacing $f$ by $-f$ also gives

$$
\sup _{\Psi}\langle T(f)\rangle_{\Psi} \geq-b+\sqrt{a^{2}+b^{2}} .
$$

Now, consider a test function $f$ with $\int f d \mu=1, f(x) \neq 0$. By lemma 2 , there exists a spacetime and a state such that $\left\langle T\left(\bar{f}_{\lambda}\right) T\left(f_{\lambda}\right)\right\rangle_{\Phi} \rightarrow+\infty$ for some appropriately chosen $f$, where $f_{\lambda}(y)=\lambda^{-D} f\left(x+\lambda^{-1}(y-x)\right)$. Hence $a \rightarrow \infty$ as $\lambda \rightarrow 0$. and therefore $\inf _{\Psi}\left\langle T\left(f_{\lambda}\right)\right\rangle_{\Psi}$ becomes arbitrarily small as $\lambda \rightarrow 0$. On the other hand $f_{\lambda} \rightarrow \delta_{x}$ in this limit, so the set $S_{x}$ is not bounded below. It similarly follows that it cannot be bounded above either.

Our next result is in some sense a converse to the above result:

Theorem 5. Let the set $S_{x}$ be equal to $\mathbb{R}$ for a given spacetime $\mathbf{M}$, and all hermitian operators $T$ not equal to a multiple of the identity. Then for any $i, k \in I$ with $i \neq \mathbf{1}$, and any sections $v_{(i)}$ of $V(i)$ we have that

$$
\operatorname{sd}\left[C_{(i)}^{(k)\left(k^{\star}\right)}\left(v_{(k)} \otimes \bar{v}_{\left(k^{\star}\right)}\right)\right]<\operatorname{sd}\left[C_{(\mathbf{1})}^{(k)\left(k^{\star}\right)}\left(v_{(k)} \otimes \bar{v}_{\left(k^{\star}\right)}\right)\right] .
$$

Remark: In generic spacetimes, we expect that the scaling degree of the right side is equal to $2 \operatorname{dim}(k)$, where $\operatorname{dim}(k)$ is the dimension of the field $\phi^{(k)}$; see the scaling degree axiom (C5). We also expect the quantity on the left side to be equal to $2 \operatorname{dim}(k)-\operatorname{dim}(i)$; see again (C5). Thus, the result tells us that, in this situation, $\operatorname{dim}(i)>0$ unless $\phi^{(i)}$ is the identity field. Thus, in this sense, the assumption of the theorem implies the asymptotic positivity axiom (C6), or-stated differently-the asymptotic positivity axiom is inconsistent with not having $S_{x}=\mathbb{R}$.

Proof: By assumption, we can find a state $\Phi$ such that $\langle T(x)\rangle_{\Phi}>A$ for each $A \in \mathbb{R}$. Consider an arbitrary, but fixed, finite collection $\phi^{(1)}, \ldots, \phi^{(n)}$ of fields. Each field is valued in some vector bundle $V(i)$. The set of expectation values of this collection of fields forms a subset which we denote

$$
K_{x}=\left\{\left(\left\langle\phi^{(1)}(x)\right\rangle_{\Phi}, \ldots,\left\langle\phi^{(n)}(x)\right\rangle_{\Phi}\right) \mid \text { states } \Phi\right\} \subset \bigoplus_{i=1}^{n} V(i)_{x}=: V_{x}
$$


Because the set of states is convex (i.e., any convex linear combination of normalized states is again a normalized state), the set $K_{x}$ is a convex subset of $V_{x}$. We claim that, in fact, $K_{x}=V_{x}$. Assume that this were not the case. Then, since any proper convex subset of a finite dimensional vector space can be enclosed by a collection of planes, there exists a collection of dual vectors $c_{(i)} \in V(i)^{\mathrm{v}}$ and an $A \in \mathbb{R}$ such that

$$
\operatorname{Re} \sum_{(i)} c_{(i)} v^{(i)}<A, \quad \text { for all }\left(v^{(1)}, \ldots, v^{(n)}\right) \in K_{x} .
$$

However, this would mean by definition that if $T=\operatorname{Re} \sum c_{(i)} \phi^{(i)}$, then $\langle T(x)\rangle_{\Phi}<A$ for all states $\Phi$, a contradiction.

Assume that the statement of the theorem is not true. Let $i=1, \ldots, n \in I$ be the field labels, with $i \neq \mathbf{1}$ for which the inequality (108) does not hold. From what we have just shown, if we define $K_{x}$ as above, then $K_{x}$ is equal to $V_{x}$. In particular, we may find states $\Phi, \Psi$, and nonzero $v^{(i)}$ with the property that

$$
\left\langle\phi^{(i)}(x)\right\rangle_{\Psi}=v^{(i)}, \quad\left\langle\phi^{(i)}(x)\right\rangle_{\Phi}=-v^{(i)} .
$$

Let $f_{\lambda}(x)=\lambda^{-D} f\left(y+\lambda^{-1}(x-y)\right)$ be a test section in the dual of the space $V(k)$, and let $\delta$ be a real number which is bigger than $2 \operatorname{dim}(k)$, but smaller than the left side of eq. (108). Using the fact that $\left\langle\phi^{(k)}\left(f_{\lambda}\right) \phi^{\left(k^{\star}\right)}\left(\bar{f}_{\lambda}\right)\right\rangle_{\Psi} \geq 0$ for all $\lambda$, we find from the operator product expansion that

$$
\lambda^{\delta} \sum_{(i)} C_{(i)}^{\left(k^{\star}\right)(k)}\left(\bar{f}_{\lambda}, f_{\lambda} ; x\right) v^{(i)} \rightarrow+\infty
$$

for at least one $f$ and a subsequence of $\lambda$ tending to 0 . Applying a similar argument to the state $\Phi$ gives that

$$
-\lambda^{\delta} \sum_{(i)} C_{(i)}^{\left(k^{\star}\right)(k)}\left(\bar{f}_{\lambda}, f_{\lambda} ; x\right) v^{(i)} \rightarrow+\infty
$$

for this subsequence of $\lambda$ tending to 0 . This is a contradiction, so the inequality (108) must hold.

\section{References}

[1] S. Axelrod and I. M. Singer, "Chern-Simons perturbation theory. 2," J. Diff. Geom. 39, 173 (1994)

[2] R. E. Borcherds, "Vertex Algebras, Kac-Moody Algebras, And The Monster," Proc. Nat. Acad. Sci. 83, 3068 (1986).

[3] H. Bostelmann, "Operator product expansions as a consequence of phase space properties,” J. Math. Phys. 46, 082304 (2005);

[4] H. Bostelmann: "Phase space properties and the short distance structure in quantum field theory," J. Math. Phys. 46, 052301 (2005) 
[5] R. Brunetti, K. Fredenhagen and M. Köhler: "The microlocal spectrum condition and Wick polynomials on curved spacetimes," Commun. Math. Phys. 180, 633-652 (1996)

[6] R. Brunetti and K. Fredenhagen: "Microlocal Analysis and Interacting Quantum Field Theories: Renormalization on physical backgrounds," Commun. Math. Phys. 208, 623-661 (2000)

[7] R. Brunetti, K. Fredenhagen and R. Verch, "The generally covariant locality principle: A new paradigm for local quantum physics," Commun. Math. Phys. 237, 31 (2003), [math-ph/0112041]; see also K. Fredenhagen, "Locally covariant quantum field theory," [arXiv:hep-th/0403007].

[8] C. J. Fewster: "Energy inequalities in quantum field theory," Proceedings of XIVth International Congress on Mathematical Physics, ed. J.-C. Zambrini, 559 (2003)

[9] K. Fredenhagen: "Locally covariant quantum field theory," Proceedings of XIVth International Congress on Mathematical Physics, ed. J.-C. Zambrini, 29 (2003)

[10] K. Fredenhagen and J. Hertel, "Local Algebras Of Observables And Point - Like Localized Fields," Commun. Math. Phys. 80, 555 (1981); K. Fredenhagen and M. Jorss, "Conformal Haag-Kastler nets, point - like localized fields and the existence of operator product expansions," Commun. Math. Phys. 176, 541 (1996).

[11] I. Frenkel, J. Lepowsky and A. Meurman, "Vertex operator algebras and the Monster," Academic Press, Boston (1988)

[12] S.A. Fulling, F.J. Narcowich, and R.M. Wald: "Singularity Structure of the TwoPoint Function in Quantum Field Theory in Curved Spacetime, II," Ann. Phys. 136 243 (1981).

[13] W. Fulton and R. MacPherson: "A compactification of configuration spaces," Ann. Math. 139183 (1994)

[14] R. Haag and D. Kastler, “An Algebraic Approach To Quantum Field Theory,” J. Math. Phys. 5, 848 (1964).

[15] S. Hollands, "The operator product expansion for perturbative quantum field theory in curved spacetime," Commun. Math. Phys. 273, 1 (2007) [arXiv:gr-qc/0605072].

[16] S. Hollands, "A general PCT theorem for the operator product expansion in curved spacetime," Commun. Math. Phys. 244, 209 (2004) [arXiv:gr-qc/0212028].

[17] S. Hollands and C. Kopper: in progress

[18] S. Hollands and R.M. Wald: in progress

[19] S. Hollands and R. M. Wald: "Local Wick Polynomials and Time Ordered Products of Quantum Fields in Curved Space," Commun. Math. Phys. 223, 289-326 (2001), [gr-qc/0103074]

[20] S. Hollands and R. M. Wald: "Existence of local covariant time-ordered-products of quantum fields in curved spacetime," Commun. Math. Phys. 231, 309-345 (2002), [gr-qc/0111108] 
[21] S. Hollands, "Quantum field theory in terms of consistency conditions I: General framework, and perturbation theory via Hochschild cohomology," arXiv:0802.2198 [hep-th].

[22] L. Hörmander: The Analysis of Linear Partial Differential Operators I, Berlin, Springer-Verlag 1983

[23] V. Kac, "Vertex algebras for beginners," Providence, USA: AMS (1996) 141 p. (University lectures series. 10)

[24] M. J. Radzikowski, "Micro-local approach to the Hadamard condition in quantum field theory on curved space-time," Commun. Math. Phys. 179, 529 (1996).

[25] V. Rivasseau, "From perturbative to constructive renormalization," Princeton, USA: Univ. Pr. (1991) 336 p. (Princeton series in physics)

[26] B. Schroer, J. A. Swieca and A. H. Volkel, "Global Operator Expansions In Conformally Invariant Relativistic Quantum Field Theory," Phys. Rev. D 11, 1509 (1975).

[27] R. F. Streater and A. A. Wightman: PCT, Spin and Statistics and All That, New York, Benjamin 1964

[28] R. M. Wald: Quantum Field Theory on Curved Spacetimes and Black Hole Thermodynamics, The University of Chicago Press, Chicago (1990)

[29] K. G. Wilson, “Nonlagrangian Models Of Current Algebra," Phys. Rev. 179, 1499 (1969).

[30] See appendix in: C. Bernard, A. Duncan, J. LoSecco, S. Weinberg: "Exact spectralfunction sum rules", Phys. Rev. D 12, 792 - 804 (1975)

[31] W. Zimmermann, "Normal Products And The Short Distance Expansion In The Perturbation Theory Of Renormalizable Interactions," Annals Phys. 77, 570 (1973) [Lect. Notes Phys. 558, 278 (2000)]. 